\title{
Critical imbeddings with multivariate rearrangements
}

\author{
by \\ Miroslav Krbec (Praha) and Hans-Jürgen Schmeisser (Jena)
}

\begin{abstract}
We are concerned with imbeddings of general spaces of Besov and Lizorkin-Triebel type with dominating mixed derivatives in the first critical case. We employ multivariate exponential Orlicz and Lorentz-Orlicz spaces as targets. We study basic properties of the target spaces, in particular, we compare them with usual exponential spaces, showing that in this case the multivariate clones are in fact better adapted to the character of smoothness of the imbedded spaces. Then we prove sharp limiting imbedding theorems and establish estimates for the multivariate growth envelope functions.
\end{abstract}

1. Introduction. Various aspects of critical imbeddings have been intensively studied in numerous papers in last years, inspired by the celebrated imbedding theorem of [Tru] and its later generalizations.

Here we shall be concerned with the limiting imbeddings of the spaces $S_{p q}^{r} B\left(\mathbb{R}^{N} \times \mathbb{R}^{N}\right)$ and $S_{p q}^{r} F\left(\mathbb{R}^{N} \times \mathbb{R}^{N}\right)$ in the so called first critical case, that is, when $r p=N$, where $2 N$ is the dimension of the underlying Euclidean space. We shall prove the refinement corresponding to the Brézis-Wainger theorem $[\mathrm{BW}]$. There is a rich literature, especially in last years, devoted to limiting imbeddings and we shall make no attempt here to give an account of either the history or the recent research in this area. We refer to [Tri2] for a detailed survey with further references.

In [KS1] and [KS2] we considered critical imbeddings of spaces with dominating mixed derivatives and various clones of special reduced imbeddings. The current paper can be considered as a free continuation of [KS1]; in [KS2] we studied imbeddings of these spaces into spaces of almost Lipschitz continuous functions (that is, in the second critical case, $r p=N+p$ ). Note

2000 Mathematics Subject Classification: Primary 46E35; Secondary 42B35, 46E30.

Key words and phrases: Sobolev spaces, Bessel potential spaces, Besov spaces, Lizorkin-Triebel spaces, exponential Orlicz spaces, Lorentz-Zygmund spaces, limiting imbeddings, dominating mixed derivatives.

The first author gratefully acknowledges the support of the Academy of Sciences of the Czech Republic, Institutional Research Plan No. AV0Z10190503, and of the grant No. 201/06/0400 of GA ČR. 
that some of these results have been quite recently generalized to the case of vector-valued function spaces without the UMD property (see e.g. [KS3]).

The target spaces for the critical imbeddings in the first critical case are exponential Orlicz spaces or their Orlicz-Lorentz refinements. In both cases the targets are characterized with the help of non-increasing rearrangements. If the imbedded spaces have a more complicated structure as in the case of dominating mixed derivatives, then there is a natural question whether the target spaces can be improved or refined by considering other spaces in the role of targets.

Recall that for a measurable $f: \mathbb{R}^{M} \rightarrow \mathbb{C}$ the non-increasing rearrangement of $f$ is the function

where

$$
f^{*}(t)=\inf \left\{\lambda>0: \mu_{f}(\lambda) \leq t\right\}
$$

$$
\mu_{f}(\lambda)=\left|\left\{x \in \mathbb{R}^{M}:|f(x)|>\lambda\right\}\right| .
$$

One of the tools used recently for refinements of subcritical Sobolev imbeddings are multivariate rearrangements (see $[\mathrm{K}]$ ), which turn out to be appropriate for our goal as well, and we shall prove the corresponding imbedding theorem in the first critical case (Theorem 3.1).

The concepts of the growth envelope function and of the growth envelope, introduced by Triebel and Haroske (see [H], [Tri2]), show that if we apply the usual rearrangements, then the use of exponential Orlicz and exponential Lorentz-Orlicz spaces in the role of the target is quite natural: the "optimal functions" permit the growth just fitting the biggest possible growth of these standard rearrangements. A closer look at the critical imbeddings in [KS1] and considerations concerning the sharpness of the imbeddings there reveal in turn that one can expect better imbeddings when passing from the usual rearrangements to multivariate ones because the latter are better adapted to measure the unboundedness of functions with dominating mixed smoothness properties.

We shall generally adhere to common notations; more specific notions will be defined. The constant $c$ in various estimates is "generic": its values might change from line to line but it is independent of the choice of functions appearing in the formulas.

\section{Rearrangements, extrapolation, spaces}

2.1. Rearrangements. Multivariate rearrangements were studied in several papers; even more general questions of Banach spaces with mixed norms were considered. For the Lebesgue and Lorentz spaces in this spirit we refer to Blozinski [B], Kolyada [K] and Yatsenko [Ya].

Let us fix notions and notations. Let $f: \mathbb{R}^{N} \times \mathbb{R}^{N} \rightarrow \mathbb{C}$ be measurable. Put 


$$
\begin{array}{ll}
\left(R_{1} f\right)(s, y)=[f(\cdot, y)]^{*}(s), & s>0, y \in \mathbb{R}^{N}, \\
\left(R_{2} f\right)(x, t)=[f(x, \cdot)]^{*}(t), & x \in \mathbb{R}^{N}, t>0,
\end{array}
$$

and define the multivariate non-increasing rearrangement of $f$ by

$$
\left(R_{12}\right) f(s, t)=\left(R_{2} \circ R_{1} f\right)(s, t)=\left[\left(R_{1} f\right)(s, \cdot)\right]^{*}(t), \quad s, t>0 .
$$

Sometimes it is more convenient to work with the averages of the above operators. Let

$$
\begin{aligned}
& \left(A_{1} f\right)(s, y)=\frac{1}{s} \int_{0}^{s}\left(R_{1} f\right)(u, y) d u, \quad s>0, y \in \mathbb{R}^{N}, \\
& \left(A_{2} f\right)(x, t)=\frac{1}{t} \int_{0}^{t}\left(R_{2} f\right)(x, v) d v, \quad x \in \mathbb{R}^{N}, t>0,
\end{aligned}
$$

and define the multivariate average of $f$ by

$$
\begin{aligned}
\left(A_{12} f\right)(s, t) & =\left(A_{2} \circ A_{1} f\right)(s, t) \\
& =\frac{1}{t} \int_{0}^{t}\left(R_{2}\left(A_{1} f\right)\right)(s, v) d v \\
& =\frac{1}{t} \int_{0}^{t} R_{2}\left(\frac{1}{s} \int_{0}^{s}\left(R_{1} f\right)(u, \cdot) d u\right)(v) d v \\
& =\frac{1}{s t} \int_{0}^{t} R_{2}\left(\int_{0}^{s}\left(R_{1} f\right)(u, \cdot) d u\right)(v) d v, \quad s, t>0 .
\end{aligned}
$$

One can consider the multivariate rearrangement $R_{21}$ and the multivariate average $A_{21}$ defined in an obvious way.

In accordance with (2.1) we should write extra parentheses around $R_{2}$ and its inner function on the third and fourth lines of the last display; since no misunderstanding can occur we shall often abuse notations a little to simplify the formulae.

On the other hand, one can consider the operator

$$
f^{* *}(s, t)=\frac{1}{s t} \int_{0}^{t s} \int_{0}^{s}\left(R_{12} f\right)(\sigma, \tau) d \sigma d \tau
$$

and ask about the relation between the various expressions above. Plainly,

$$
\left(R_{12} f\right)(s, t) \leq\left(A_{12} f\right)(s, t) \leq f^{* *}(s, t) .
$$

By the Fubini theorem and properties of non-increasing rearrangements, the function $R_{12} f$ is equimeasurable with $R_{1} f$ and the latter is in turn 
equimeasurable with $f$. Hence

$$
\begin{aligned}
\int_{0}^{\infty} \int_{0}^{\infty}\left(R_{12}\right) f(s, t) d s d t & =\int_{\mathbb{R}^{N}} \int_{\mathbb{R}^{N}} f(x, y) d x d y \\
& =\int_{0}^{\infty} R_{1}\left(\int_{0}^{\infty}\left(R_{2} f\right)(x, \cdot) d t\right)(s) d s .
\end{aligned}
$$

An analogous equality holds for $R_{21}$.

The superposition of the rearrangements in (2.2) and of the averages in (2.3) is worth a comment. It should be pointed out that the functions $R_{12} f$ and $A_{12} f$ are non-increasing with respect to both variables. This means the following: If for a fixed $x$ we rearrange a function $f=f(x, y)$ with respect to $y$, then the result is the non-increasing function $t \mapsto\left(R_{2} f\right)(x, t), t>0$. Now, if we rearrange $\left(R_{2} f\right)(x, t)$ as a function of $x$ for every fixed $t$, it can generally happen that the resulting function will be rearranged in a different way in the direction of the $t$-axis; nevertheless, the monotonicity in $t$ will be preserved! This remarkable property of multivariate rearrangements can be seen and understood properly for instance by considering a rearrangement of a matrix, where the application of $R_{12}$ consists in subsequent non-increasing rearrangements of the elements in columns and rows. Hence

$$
R_{2}\left(R_{12} f\right)(s, t)=R_{1}\left(R_{12} f\right)(s, t)=\left(R_{12} f\right)(s, t), \quad s, t>0 .
$$

Further, one can easily check that

$$
\left(R_{12}(f+g)\right)\left(s_{1}+s_{2}, t_{1}+t_{2}\right) \leq\left(R_{12} f\right)\left(s_{1}, t_{1}\right)+\left(R_{12} g\right)\left(s_{2}, t_{2}\right) .
$$

The operator $A_{12}$ is subadditive whereas $R_{12}$ and $f^{* *}$ are not.

The symbol $L^{p, q}(1 \leq p, q \leq \infty)$ will denote the usual Lorentz space equipped with the (quasi)norm

$$
\left\|f \mid L^{p, q}\right\|=\left(\frac{q}{p} \int_{0}^{\infty}\left[t^{1 / p} f^{*}(t)\right]^{q} \frac{d t}{t}\right)^{1 / q}
$$

(with the usual modifications if $p=\infty$ or $q=\infty$ ).

The multivariate Lorentz spaces can be introduced as follows: Consider for simplicity the product $\mathbb{R}^{2 N}=\mathbb{R}^{N} \times \mathbb{R}^{N}$. Then for $1<p, q<\infty$ we define the (quasi)norm in $L_{R}^{p, q}=L_{R}^{p, q}\left(\mathbb{R}^{N} \times \mathbb{R}^{N}\right)$ by

$$
\left\|f \mid L_{R}^{p, q}\right\|=\left(\frac{q}{p} \int_{0}^{\infty} \frac{q}{p} \int_{0}^{\infty}\left[(s t)^{1 / p}\left(R_{12} f\right)(s, t)\right]^{q} \frac{d s}{s} \frac{d t}{t}\right)^{1 / q} .
$$

Similarly one can use $R_{21}$. Relations between $L_{R}^{p, q}$ and the standard Lorentz spaces $L^{p, q}$ were studied in [Ya] where it was shown, for instance, that

$$
L_{R}^{p, q} \hookrightarrow L^{p, q} \quad \text { if } q<p .
$$


The domain $\mathbb{R}^{N} \times \mathbb{R}^{N}$ will be considered to simplify the notations and the exposition. We shall not pursue the technicalities connected with the more general domain $\mathbb{R}^{N_{1}} \times \mathbb{R}^{N_{2}}$ and the corresponding spaces with mixed norms.

Let us recall the sharp form of Calderón's lemma (see [SW, Chapter 5]), stating that if $p \geq q$, then $\left\|f\left|L_{p}\|\leq\| f\right| L^{p, q}\right\|$, i.e. the norm of the corresponding imbedding does not exceed one. This combined with (2.8) and with the equimeasurability properties (2.5) immediately yields

$$
\left\|f\left|L_{p}\left(\mathbb{R}^{2 N}\right)\|\leq c\| f\right| L_{R}^{p, q}\left(\mathbb{R}^{N} \times \mathbb{R}^{N}\right)\right\| \quad \text { if } q<p,
$$

where $c$ is the norm of the imbedding in (2.8). Applying the sharp version of Calderón's lemma in a more careful and elaborate way one can improve the last estimate to

$$
\left\|f\left|L_{p}\left(\mathbb{R}^{2 N}\right)\|\leq\| f\right| L_{R}^{p, q}\left(\mathbb{R}^{N} \times \mathbb{R}^{N}\right)\right\| \quad \text { if } q<p,
$$

that is, the norm of the imbedding $L_{R}^{p, q}\left(\mathbb{R}^{N} \times \mathbb{R}^{N}\right) \hookrightarrow L_{p}\left(\mathbb{R}^{2 N}\right)$ does not exceed one. Indeed, let $f=f(x, y), x, y \in \mathbb{R}^{N}$, and $q<p$. Then

$$
\begin{aligned}
\left\|f \mid L_{p}\left(\mathbb{R}^{N} \times \mathbb{R}^{N}\right)\right\| & \leq\left\|\left(\frac{q}{p} \int_{0}^{\infty}\left[s^{1 / p} R_{1} f(s, y)\right]^{q} \frac{d s}{s}\right)^{1 / q} \mid L_{p}\left(\mathbb{R}^{N}\right)\right\| \\
& =\left\|\frac{q}{p} \int_{0}^{\infty}\left[s^{1 / p} R_{1} f(s, y)\right]^{q} \frac{d s}{s} \mid L_{p / q}\left(\mathbb{R}^{N}\right)\right\|^{1 / q} \\
& \leq\left(\frac{q}{p} \int_{0}^{\infty}\left(\int_{\mathbb{R}^{N}}\left[s^{1 / p} R_{1} f(s, y)\right]^{p} d y\right)^{q / p} \frac{d s}{s}\right)^{1 / q} \\
& =\left(\frac{q}{p} \int_{0}^{\infty}\left\|s^{1 / p} R_{1} f(s, \cdot) \mid L_{p}\left(\mathbb{R}^{N}\right)\right\|^{q} \frac{d s}{s}\right)^{1 / q} \\
& \leq\left(\frac{q}{p} \int_{0}^{\infty}\left(\frac{q}{p} \int_{0}^{\infty}\left[s^{1 / p} R_{12} f(s, t) t^{1 / p}\right]^{q} \frac{d t}{t}\right) \frac{d s}{s}\right)^{1 / q} \\
& =\left\|f \mid L_{R}^{p, q}\left(\mathbb{R}^{N} \times \mathbb{R}^{N}\right)\right\|, \quad \text { (by Calderón's lemma) }
\end{aligned}
$$

and (2.9) is proved.

Since we shall work with atomic decompositions it will be useful to introduce further shorthands. Let $b: \mathbb{Z}^{N} \times \mathbb{Z}^{N} \rightarrow \mathbb{C}$ and denote the values of $b$ by $b(m, n)$. Put

$$
\begin{aligned}
\left(R_{1} b\right)(\alpha, n) & =[b(\cdot, n)]^{*}(\alpha), \quad \alpha \in \mathbb{N}, n \in \mathbb{Z}^{N}, \\
\left(R_{2} b\right)(m, \beta) & =[b(m, \cdot)]^{*}(\beta), \quad m \in \mathbb{Z}^{N}, \beta \in \mathbb{N},
\end{aligned}
$$




$$
\begin{aligned}
\left(R_{12} b\right)(\alpha, \beta) & =\left(R_{2} \circ R_{1} b\right)(\alpha, \beta)=\left[\left(R_{1} b\right)(\alpha, \cdot)\right]^{*}(\beta), \quad \alpha, \beta \in \mathbb{N}, \\
\left(A_{1} b\right)(\mu, n) & =\frac{1}{\mu} \sum_{\alpha=1}^{\mu}\left(R_{1} b\right)(\alpha, n), \quad \mu \in \mathbb{N}, n \in \mathbb{Z}^{N}, \\
\left(A_{2} b\right)(m, \nu) & =\frac{1}{\nu} \sum_{\beta=1}^{\nu}\left(R_{2} b\right)(m, \beta), \quad m \in \mathbb{Z}^{N}, \nu \in \mathbb{N}, \\
b^{* *}(\mu, \nu) & =\frac{1}{\mu \nu} \sum_{\alpha=1}^{\mu} \sum_{\beta=1}^{\nu}\left(R_{12} b\right)(\alpha, \beta), \quad \mu \in \mathbb{N}, \nu \in \mathbb{N} .
\end{aligned}
$$

Next we collect a couple of inequalities which will be useful in what follows. The inequalities in the following lemma are standard and can be found e.g. in the classical monograph [HLP].

Lemma 2.1 (Hardy's discrete inequality). Let $1<q \leq \infty$ and let $\left(b_{\kappa}\right)$ and $\left(b_{\kappa, \lambda}\right), \kappa, \lambda \in \mathbb{N}$, be sequences of non-negative real numbers. Then

$$
\sum_{k=1}^{\infty}\left(\frac{1}{k} \sum_{\kappa=1}^{k} b_{\kappa}\right)^{q} \leq c \sum_{\kappa=1}^{\infty} b_{\kappa}^{q}
$$

and

$$
\sum_{k=1}^{\infty} \sum_{l=1}^{\infty}\left(\frac{1}{k l} \sum_{\kappa=1}^{k} \sum_{\lambda=1}^{l} b_{\kappa, \lambda}\right)^{q} \leq c \sum_{\kappa=1}^{\infty} \sum_{\lambda=1}^{\infty} b_{\kappa, \lambda}^{q}
$$

(with an appropriate modification for $q=\infty$ ).

A consequence of this lemma is the following maximal inequality, which holds for any sequence $b=\left(b^{m, n}\right), m, n \in \mathbb{Z}^{N}$ :

$$
\sum_{\mu=1}^{\infty} \sum_{\nu=1}^{\infty}\left[b^{* *}(\mu, \nu)\right]^{q} \leq c \sum_{\alpha=1}^{\infty} \sum_{\beta=1}^{\infty}\left(R_{12} b\right)(\alpha, \beta)^{q}=c \sum_{m \in \mathbb{Z}^{N}} \sum_{n \in \mathbb{Z}^{N}}\left|b^{m, n}\right|^{q} .
$$

2.2. Extrapolation. In the following we shall need various refinements of Orlicz spaces. To unify terminology and notations we give a short survey and several definitions. To avoid unnecessary technicalities (arising from considering spaces on sets with infinite measure) we shall tacitly assume that the spaces below are defined on some open set $\Omega$ in $\mathbb{R}^{N}$ with $|\Omega|=1$. We shall suppose that the reader is familiar with Orlicz spaces $L^{\Phi}$, where $\Phi$ is a Young function. Important in applications are the Zygmund spaces, $L^{p}(\log L)^{a}$, i.e. the Orlicz spaces with the generating Young function $\Phi(t)=t^{p}(\log (1+t))^{a}$, $1 \leq p<\infty, a \in \mathbb{R}$. Note that the Orlicz spaces are rearrangement invariant and that $f \in L^{p}(\log L)^{a}$ is equivalent to $\int_{0}^{1}\left[f^{*}(t)\right]^{p}(\log e / t)^{a} d t<\infty$ (see e.g. [BR] or [BS]). Hence if $p=\infty$, it is well justified to consider $L^{\infty}(\log L)^{a}$ 
$(a<0)$ as the space with the finite quasinorm $\sup _{0<t<1} f^{*}(t)(\log e / t)^{1 / a}$. This also naturally agrees with the extrapolation properties (2.11) and (2.12) below so that in this case we get the exponential Orlicz space $L^{\exp t^{-a}}$.

In [BR] the so called Lorentz-Zygmund spaces $L^{p, q ; a}$ were studied, for $1 \leq p, q \leq \infty, a \in \mathbb{R}$, which-in these notations-include the Zygmund spaces $L^{p}(\log L)^{a}(1 \leq p<\infty)$, the exponential Orlicz spaces $L^{\exp t^{a}}, a>0$ (which are just the Zygmund spaces $\left.L^{\infty}(\log L)^{-1 / a}\right)$ and also, for $a=0$, the Lorentz spaces $L^{p, q}$. The general formula for the (quasi)norm in the space $L^{p, q ; a}, 1 \leq p, q \leq \infty$, reads

$$
\left\|f \mid L^{p, q ; a}\right\|=\left(\frac{q}{p} \int_{0}^{1}\left[t^{1 / p} f^{*}(t)(\log e / t)^{a}\right]^{q} d t / t\right)^{1 / q}
$$

with appropriate modifications for $p=\infty$ or $q=\infty$ : If $1 \leq p<\infty$ and $q=$ $\infty$, we get the weak spaces with the quasinorm $\sup _{0<t \leq 1} t^{1 / p} f^{*}(t)(\log e / t)^{a}$. If $p=\infty, 1 \leq q<\infty$, and $a \neq 0$, then we have the space $L^{\infty, q ; a}$ with the norm

$$
\left(\int_{0}^{1}\left[f^{*}(t)(\log e / t)^{a}\right]^{q} d t / t\right)^{1 / q}
$$

The case $a=-1$ will be of particular importance for us; this is the case of the exponential Orlicz-Lorentz space, sometimes called the $B W_{q^{-}}$space because of its prominent role in critical imbeddings - we refer to the celebrated paper [BW]. See also comments later in this section.

To have a more complete picture we recall several facts about extrapolation of Lebesgue and Lorentz spaces. It is a "folklore" fact that the space $L^{\exp t^{q}}$, i.e. the Orlicz space with the generating Young function $t \mapsto$ $\exp t^{q}-1$, can be characterized as the space of all functions $f$ which satisfy

$$
\sup _{k \in \mathbb{N}} \frac{\left\|f \mid L_{k}\right\|}{k^{1 / q}}<\infty
$$

or

$$
\sup _{0<t<1} \frac{f^{*}(t)}{(\log e / t)^{1 / q}}<\infty
$$

or

$$
\sup _{0<t<1} \frac{\left\|f \mid L^{k, \infty}\right\|}{k^{1 / q}}<\infty
$$

(further refinements in terms of Lorentz spaces $L^{k, r}$ are possible - see e.g. $[\mathrm{EK}])$. Let us point out that all the three formulas above give equivalent (quasi)norms.

On the other hand, Lorentz type clones of Orlicz spaces, $L^{\Phi, \Psi}$ in selfexplaining notations ( $\Psi$ and $\Phi$ are Young functions), have been studied in a number of papers (see e.g. [M-S]). If $\Phi(t)=\exp t^{q^{\prime}}-1, \Psi(t)=t^{q}$, 
$q^{\prime}=q /(q-1)$ with $1<q<\infty$ (see [EK] for an analysis of this case), then $L^{\exp t^{q^{\prime}}-1, t^{q}}$ (or just $L^{\exp t^{q^{\prime}}, t^{q}}$ for brevity) can be shown to coincide with the Lorentz-Zygmund spaces $L^{\infty, q,-1}$ with the quasinorm

$$
\left(\int_{0}^{1}\left[\frac{f^{*}(t)}{\log e / t}\right]^{q} \frac{d t}{t}\right)^{1 / q} .
$$

Let us point out that these spaces are nothing else than $B W_{q}$ of $[\mathrm{BR}]$ mentioned earlier.

Discretizing the integral on the left (e.g. using dyadic intervals) leads to various extrapolation characterizations of these spaces; see [CUK]. Note in passing that the space $L^{\exp t^{q^{\prime}}, t^{q}}, 1<q<\infty$, can be characterized by the condition

$$
\left(\sum_{k=1}^{\infty}\left[\frac{\left\|f \mid L_{k}\right\|}{k}\right]^{q}\right)^{1 / q}<\infty,
$$

and that another equivalent (quasi)norm in $L^{\exp t^{q^{\prime}}, t^{q}}$ can be taken as

$$
\left(\sum_{k=1}^{\infty}\left[\frac{\left\|f \mid L^{k, r}\right\|}{k}\right]^{q}\right)^{1 / q}
$$

with any $1<r<\infty$. We refer to [EK] and [CUK]) for a systematic treatment of these and "localized" blowup conditions for Lebesgue and Lorentz norms.

In the current paper we deal with a multivariate variant of (2.10) and (2.13). Quasinorms of this type will be used to handle the critical imbeddings - see e.g. (3.1) in Theorem 3.1. Although our main objective is an improvement of critical imbeddings and we shall not systematically study the corresponding multivariate spaces themselves, we prove various relevant comparisons with the spaces based on usual rearrangements (in this section and later in Sections 4 and 5).

Let us also observe that the condition (3.1) expresses an imbedding between the appropriate spaces provided all functions live in a bounded extension domain; generally it should be understood as a measurement of the large values of $|f|$ in terms of integrability properties of its rearrangement near the origin.

Note also that Theorem 3.1 can be viewed as a certain multivariate Lorentz refinement of the critical imbeddings in [KS1], in the same spirit as the Sobolev space $W^{k, p}$ in the sublimiting case is imbedded into the Lorentz space $L^{p^{*}, p}$, where $p^{*}=N p /(N-k p)$ is the Sobolev exponent, that is, into a "better" space than the standard target $L_{p^{*}}$.

To be more specific, we shall consider the multivariate Lorentz-Zygmund spaces $L_{R}^{\infty q}(\log L)^{-a}(\log L)^{-b}=L_{R}^{\infty q}(\log L)^{-a}(\log L)^{-b}\left(\Omega_{1} \times \Omega_{2}\right)$, where 
$\Omega_{1} \times \Omega_{2} \subset \mathbb{R}^{N} \times \mathbb{R}^{N},\left|\Omega_{1}\right|=\left|\Omega_{2}\right|=1$, consisting of functions $f$ with the finite quasinorm

$$
\begin{aligned}
\left\|f \mid L^{\infty q}(\log L)^{-a}(\log L)^{-b}\right\| & \\
= & \left(\int_{0}^{1} \int_{0}^{1}\left[\frac{\left(R_{12} f\right)(s, t)}{(\log e / s)^{a}(\log e / t)^{b}}\right]^{q} \frac{d s}{s} \frac{d t}{t}\right)^{1 / q}
\end{aligned}
$$

in the case adapted to our splitting $\mathbb{R}^{2 N}=\mathbb{R}^{N} \times \mathbb{R}^{N}$. Let us observe that in the same spirit as in [M-S] and $[\mathrm{EK}]$ one can introduce and consider more general multivariate Lorentz-Orlicz spaces $L_{R}^{\left(\Phi_{1}, \Psi_{1}\right),\left(\Phi_{2}, \Psi_{2}\right)}$, the special case $L_{R}^{\left(\exp t^{q^{\prime}}, t^{q}\right),\left(\exp t^{q^{\prime}}, t^{q}\right)}$ of which would correspond to (2.15) with $a=b=1$ (cf. the comments before (2.13)).

If $q=\infty$, then we have the space $L_{R}^{\infty, \infty}(\log L)^{-a}(\log L)^{-b}$ for $a, b \neq 0$, with the quasinorm

$$
\sup _{0<s, t \leq 1} \frac{\left(R_{12} f\right)(s, t)}{(\log e / s)^{a}(\log e / t)^{b}}
$$

Proposition 2.2. Let $1<q<\infty, a, b>0, \Omega_{1}, \Omega_{2} \subset \mathbb{R}^{N},\left|\Omega_{1}\right|=\left|\Omega_{2}\right|=1$, and let $f$ be a measurable function in $\Omega_{1} \times \Omega_{2}$. Then

$$
\begin{aligned}
\sum_{k=1}^{\infty}\left[\frac{\left\|f \mid L_{k}\left(\Omega_{1} \times \Omega_{2}\right)\right\|}{k^{a+b}}\right]^{q} & \leq c \sum_{i=1}^{\infty} \sum_{j=1}^{\infty}\left[\frac{\left(R_{12} f\right)\left(2^{-i}, 2^{-j}\right)}{i^{a} j^{b}}\right]^{q} \\
& \leq c \int_{0}^{1} \int_{0}^{1}\left[\frac{\left(R_{12} f\right)(s, t)}{(\log e / s)^{a}(\log e / t)^{b}}\right]^{q} \frac{d s}{s} \frac{d t}{t} .
\end{aligned}
$$

Proof. Let $k \geq q$. Then $L^{k, q} \hookrightarrow L_{k}$ with the norm of the imbedding not exceeding one (see [SW, Chapter V, Theorem 3.11]).

It follows from (2.9) and (2.7) that

$$
\begin{aligned}
\| f \mid L_{k}\left(\Omega_{1}\right. & \left.\times \Omega_{2}\right) \|^{q} \leq \frac{q^{2}}{k^{2}} \int_{0}^{1} \int_{0}^{1}\left[(s t)^{1 / k}\left(R_{12} f\right)(s, t)\right]^{q} \frac{d s}{s} \frac{d t}{t} \\
& \leq \frac{c q^{2}}{k^{2}} \sum_{i=0}^{\infty} \sum_{j=0}^{\infty} \int_{2^{-j-1}}^{2^{-j}} \int_{2^{-i-1}}^{2^{-i}}\left[2^{-i / k} 2^{-j / k}\left(R_{12} f\right)\left(2^{-i-1}, 2^{-j-1}\right)\right]^{q} \frac{d s}{s} \frac{d t}{t} \\
& \leq \frac{c q^{2}}{k^{2}} \sum_{i=0}^{\infty} \sum_{j=0}^{\infty} 2^{-q i / k} 2^{-q j / k}\left[\left(R_{12} f\right)\left(2^{-i-1}, 2^{-j-1}\right)\right]^{q}
\end{aligned}
$$


Hence

$$
\begin{aligned}
& \sum_{k=[q]+1}^{\infty}\left[\frac{\left\|f \mid L_{k}\left(\Omega_{1} \times \Omega_{2}\right)\right\|}{k^{a+b}}\right]^{q} \\
& \leq c q^{2} \sum_{i=0}^{\infty} \sum_{j=0}^{\infty}\left[\left(R_{12} f\right)\left(2^{-i-1}, 2^{-j-1}\right)\right]^{q} \sum_{k=[q]+1}^{\infty}\left[k^{-a q-1} 2^{-q i / k}\right]\left[k^{-b q-1} 2^{-q j / k}\right] .
\end{aligned}
$$

Denote the last sum by $S(a, b, q)$; then

$$
S(a, b, q) \leq\left(\sum_{k=[q]+1}^{\infty} k^{-a q-1} 2^{-q i / k}\right)\left(\sum_{k=[q]+1}^{\infty} k^{-b q-1} 2^{-q j / k}\right) .
$$

Replacing the first sum on the right hand side by an integral and changing variables, we get, for $i>0$,

$$
\begin{aligned}
\sum_{k=1}^{\infty} k^{-b q-1} 2^{-q i / k} & \sim \int_{1}^{\infty} \xi^{-a q} \exp \left(-\frac{q i}{\xi} \log 2\right) \frac{d \xi}{\xi} \\
& \sim \int_{0}^{q i \log 2} \xi^{a q-1}\left(\frac{1}{q i \log 2}\right)^{a q} e^{-\xi} d \xi \\
& =\left(\frac{1}{q i \log 2}\right)^{a q} \Gamma(a q) \leq c i^{-a q},
\end{aligned}
$$

where $\Gamma$ denotes the Gamma function. An analogous estimate holds true for the second sum. Hence we get, for $i, j>0$,

$$
S(a, b, q) \leq c i^{-a q} j^{-b q},
$$

which proves the first inequality in (2.16).

To prove the right inequality in $(2.16)$ consider

$$
2^{-k-1} \leq s \leq 2^{-k} \quad \text { and } \quad 2^{-l-1} \leq t \leq 2^{-l}
$$

By the monotonicity properties of $R_{1,2} f$ and of log we have

$$
\int_{2^{-k-1}}^{2^{-k}} \int_{2^{-l-1}}^{2^{-l}}\left[\frac{\left(R_{12} f\right)(s, t)}{(\log e / s)^{a}(\log e / t)^{b}}\right]^{q} \frac{d s}{s} \frac{d t}{t} \geq c\left[\frac{\left(R_{12} f\right)\left(2^{-k}, 2^{-l}\right)}{k^{a} l^{b}}\right]^{q},
$$

and the assertion follows by summing over $k$ and $l$.

Corollary 2.3. Let the assumptions on $\Omega_{j}, j=1,2$, and $f$ from the previous proposition be satisfied. Then

$$
\int_{0}^{1}\left[\frac{f^{*}(t)}{(\log e / t)^{2}}\right]^{r} \frac{d t}{t} \leq c \int_{0}^{1} \int_{0}^{1}\left[\frac{\left(R_{12} f\right)(s, t)}{(\log e / s)(\log e / t)}\right]^{r} \frac{d s}{s} \frac{d t}{t}
$$

for any $1<r<\infty$. 
Proof. Discretizing the integral on the left hand side of (2.17) we can write

$$
\begin{aligned}
\int_{0}^{1}\left[\frac{f^{*}(t)}{(\log e / t)^{2}}\right]^{r} \frac{d t}{t} & \leq c \sum_{k=1}^{\infty} \frac{f^{*}\left(2^{-k}\right)^{r}}{k^{2 r}} \leq c \sum_{k=1}^{\infty} \frac{1}{k^{2 r}}\left(\int_{2^{-k+1}}^{2^{-k}} f^{*}(t)^{k} d t\right)^{r / k} \\
& \leq c \sum_{k=1}^{\infty}\left[\frac{\left\|f \mid L_{k}\right\|}{k^{2}}\right]^{r}
\end{aligned}
$$

Now (2.17) follows on invoking Proposition 2.2 with $a=b=1$.

REMARK 2.4. The left hand side of (2.18) equals $\left\|f \mid L^{\infty, r ;-2}\right\|^{r}$ (see (2.10)). The above proof shows that $\left\|f \mid L^{\infty, r ;-2}\right\|$ can be estimated from above by a series similar to (2.14) (with $k$ in the denominator replaced by $k^{2}$ ). One can prove that the converse estimate also holds, hence there is in fact an equivalence. An analogous equivalence can be proved for all the spaces $\left\|f \mid L^{\infty, r ;-a}\right\|(1<r<\infty, a>0)$. We omit the details and refer to [CUK] for the idea of the proof.

Proposition 2.5. Let the assumptions on $\Omega_{j}, j=1,2, a, b$, and $f$ from the previous proposition be satisfied. Then

$$
\sup _{k} \frac{\left\|f \mid L_{k}\left(\Omega_{1} \times \Omega_{2}\right)\right\|}{k^{a+b}} \leq c \sup _{0<s<1} \sup _{0<t<1} \frac{\left(R_{12} f\right)(s, t)}{(\log e / s)^{a}(\log e / t)^{b}} .
$$

Proof. Let the right hand side of (2.19) be finite, otherwise there is nothing to prove. Then

$$
\left(R_{12} f\right)(s, t) \leq \gamma\left(\log \frac{e}{s}\right)^{a}\left(\log \frac{e}{t}\right)^{b}
$$

for some constant $\gamma>0$. After integration (because $f$ is equimeasurable with $R_{12} f$ ),

$$
\begin{aligned}
\left\|f \mid L_{k}\left(\Omega_{1} \times \Omega_{2}\right)\right\| & =\left(\int_{0}^{1} \int_{0}^{1}\left[\left(R_{12} f\right)(s, t)\right]^{k} d s d t\right)^{1 / k} \\
& \leq \gamma\left(\int_{0}^{1}\left[\log \frac{e}{s}\right]^{a k} d s\right)^{1 / k}\left(\int_{0}^{1}\left[\log \frac{e}{t}\right]^{b k} d t\right)^{1 / k} .
\end{aligned}
$$

The integrals with the logarithmic function can be estimated as follows. We have, after changes of variables, $\int_{0}^{1}\left[\log \frac{e}{s}\right]^{a k} d s=e \int_{e}^{\infty}(\log x)^{a k} \frac{d x}{x^{2}}=e \int_{1}^{\infty} y^{a k} e^{-y} d y \leq e \Gamma(a k+1)=e a k \Gamma(a k)$. 
Hence

$$
\left(\int_{0}^{1}\left[\log \frac{e}{s}\right]^{a k} d s\right)^{1 / k} \leq(e a k \Gamma(a k))^{1 / k} \leq c(\Gamma(a k))^{1 / k} .
$$

By Stirling's formula,

$$
\Gamma(a k)^{1 / k} \leq\left[(a k)^{a k-1 / 2} e^{-a k}\right]^{1 / k} \leq c k^{a-1 /(2 k)} \leq c k^{a} .
$$

This gives

$$
\left(\int_{0}^{1}\left[\log \frac{e}{s}\right]^{a k} d s\right)^{1 / k} \leq c k^{a}
$$

so that

$$
\left\|f \mid L_{k}\left(\Omega_{1} \times \Omega_{2}\right)\right\| \leq c \gamma k^{a+b} .
$$

Next we compare the usual exponential spaces with their multivariate "diagonal case".

Corollary 2.6. Under the assumptions of Proposition 2.2,

$$
\sup _{0<t \leq 1} \frac{f^{*}(t)}{(\log e / t)^{2 a}} \leq c \sup _{0<t \leq 1} \sup _{0<s \leq 1} \frac{\left(R_{12} f\right)(s, t)}{(\log e / s)^{a}(\log e / t)^{a}} .
$$

Proof. It is enough to use the fact that the left hand side of (2.20) is equivalent to the left hand side of (2.19) when $a=b$ (see (2.11) and (2.12)); note for completeness that the left hand side of (2.20) is equivalent to $\left\|f \mid L^{\exp t^{1 /(2 a)}}\right\|$.

REMARK 2.7. It is possible to give an alternative proof of (2.20) without invoking the equivalence of various extrapolation characterizations of the exponential Orlicz spaces in question. Indeed, let

$$
\varphi(s, t)=(\log e / s)(\log e / t), \quad s, t>0,
$$

and assume that the right hand side of (2.20) is finite. Then

$$
\left(R_{12} f\right)(s, t) \leq \gamma[\varphi(s, t)]^{a}
$$

with some $0<\gamma<\infty$. However, it is not difficult to show that

$$
\varphi^{*}(\tau) \sim(\log e / \tau)^{2} .
$$

Since $f$ and $R_{12} f$ are equimeasurable it follows that

$$
f^{*}(\tau)=\left(R_{12} f\right)^{*}(\tau) \leq \gamma\left[\varphi^{*}(\tau)\right]^{a} \leq c_{1}(\log e / \tau)^{2 a},
$$

which proves the corollary.

REMARK 2.8. Denote, for brevity, by $Q_{\infty, a, b}(f)$ the right hand side of (2.19), that is, $\left\|f \mid L_{R}^{\infty, \infty}(\log L)^{-a}(\log L)^{-b}\right\|$. Then

$$
Q_{\infty, a, b}(f) \sim\|\| f\left|L^{\exp t^{1 / b}}(y)\left\|\mid L^{\exp t^{1 / a}}(x)\right\|,\right.
$$


where on the right hand side there is a mixed norm of the function $f=$ $f(x, y)$, first taken with respect to $y$ and then with respect to $x$ (which is indicated by the occurrence of the variable in the symbol for the space). Because of the equimeasurability one can alternatively take $R_{2}$ in the inner norm, the rearrangement $R_{1}$ of it, and finally the norm with respect to the variable $x$. In other words, $Q_{\infty, a, b}(f)$ can be viewed as a certain "mixed iterated quasinorm".

Indeed,

$$
\begin{aligned}
\frac{\left(R_{12} f\right)(s, t)}{(\log e / s)^{a}(\log e / t)^{b}} & =\frac{1}{(\log e / s)^{a}} R_{1}\left(\frac{\left(R_{2} f\right)(x, \cdot)(t)}{\log e / t)^{b}}\right)(s) \\
& \leq \frac{1}{(\log e / s)^{a}} R_{1}\left(\sup _{0<t \leq 1} \frac{\left(R_{2} f\right)(x, \cdot)(t)}{(\log e / t)^{b}}\right)(s) \\
& \leq \frac{c}{(\log e / s)^{a}} R_{1}\left(\left\|f(x, \cdot) \mid L^{\exp t^{1 / b}}\right\|\right)(s) \\
& \leq c \sup _{0<s \leq 1} \frac{1}{(\log e / s)^{a}} R_{1}\left(\left\|f(x, \cdot) \mid L^{\exp t^{1 / b}}\right\|\right)(s) \\
& \leq c\|\| f(x, \cdot)\left|L^{\exp t^{1 / b}}\left\|\mid L^{\exp s^{1 / a}}\right\| .\right.
\end{aligned}
$$

On the other hand, there exists $c>0$ such that for every $x$ there exists $t=t_{x}$ such that

$$
\frac{\left(R_{2} f\right)\left(x, t_{x}\right)}{\left(\log e / t_{x}\right)^{b}} \geq \frac{c}{2}\left\|R_{2} f(x, \cdot) \mid L^{\exp t^{1 / b}}\right\|
$$

Hence also

$$
\begin{aligned}
\sup _{0<s \leq 1} \frac{1}{(\log e / s)^{a}} R_{1} & \left(\sup _{0<t \leq 1} \frac{\left(R_{2} f\right)(x, t)}{(\log e / t)^{b}}\right)(s) \\
& \geq \sup _{0<s \leq 1} \frac{1}{(\log e / s)^{a}} R_{1}\left(\frac{c}{2}\left\|R_{2} f(x, \cdot) \mid L^{\exp t^{1 / b}}\right\|\right)(s) \\
& \geq \frac{c^{2}}{4} Q_{\infty, a, b}(f) .
\end{aligned}
$$

The inequality (2.20) can therefore be understood as a comparison of of the usual Orlicz exponential space with the diagonal case of the mixed norm space with the multivariate rearrangements, the latter space being nothing else than a special (diagonal) case of a more general exponential mixed norm space. Hence there is a natural question whether the diagonal case gives the usual norm, i.e. whether there is an equivalence in (2.20). Rather surprisingly, this is not true. Let us give a simple example. For sake of clarity let us temporarily use the variable in the symbols for the spaces. Let $N=1$, $a=1$, and consider $g=g(y), 0<y \leq 1$, such that $\left\|g \mid L_{k}(y)\right\| \sim k^{2}, k \in \mathbb{N}$. Then $g \notin L^{\exp t}(y)$; equivalently $\sup _{k}\left\|g \mid L_{k}(y)\right\| / k=\infty$. Put 


$$
f(x, y)=g(y), \quad 0<x, y \leq 1
$$

Plainly,

$$
\left\|f \mid L_{k}(x, y)\right\| \sim k^{2}
$$

hence the left hand side of (2.20) is finite whereas the right hand side is infinite.

Similar analysis can be carried out for other estimates in this section; as agreed earlier, our major concern in this paper will be the critical imbeddings.

In the last two assertions we shall compare the multivariate expressions of the exponential Orlicz and Lorentz-Orlicz type (cf. [Tri2, Section 12]).

Proposition 2.9. Let $1<q<\infty$ and $a, b>0$. Then

$$
\begin{aligned}
& \sup _{0<s<1} \sup _{0<t<1} \frac{\left(R_{12} f\right)(s, t)}{(\log e / s)^{a}(\log e / t)^{b}} \\
& \leq c\left(\int_{0}^{1} \int_{0}^{1}\left[\frac{\left(R_{12} f\right)(s, t)}{(\log e / s)^{a}(\log e / t)^{b}}\right]^{q} \frac{d s}{s \log e / s} \frac{d t}{t \log e / t}\right)^{1 / q} .
\end{aligned}
$$

Proof. We have

$$
\left(\log \frac{e}{s}\right)^{-a q}=-a q\left(\log \frac{e}{s}\right)^{-a q-1}\left(-\frac{e}{s}\right)=e a q \frac{1}{(\log e / s)^{a q}} \frac{1}{s \log e / s} .
$$

Thus

$$
\left(\log \frac{e}{s}\right)^{-a q} \leq e a q \int_{0}^{s} \frac{1}{(\log e / u)^{a q}} \frac{d u}{u \log e / u}
$$

and

$$
\begin{aligned}
& \left(\log \frac{e}{s}\right)^{-a q}\left(\log \frac{e}{t}\right)^{-b q} \\
& \quad \leq e^{2} a b q^{2} \int_{0}^{s t} \frac{1}{(\log e / u)^{a q}(\log e / v)^{b q}} \frac{d u}{u \log e / u} \frac{d v}{v \log e / v}
\end{aligned}
$$

Since $R_{12} f$ is non-increasing with respect to both variables, this implies

$$
\begin{aligned}
& \frac{\left(R_{12} f\right)(s, t)}{(\log e / s)^{a}(\log e / t)^{b}} \\
& \leq c\left(\int_{00}^{s} \int_{0}^{t}\left[\frac{\left(R_{12} f\right)(u, v)}{(\log e / u)^{a}(\log e / v)^{b}}\right]^{q} \frac{d u}{u \log e / u} \frac{d v}{v \log (e / v}\right)^{1 / q} \\
& \leq c\left(\int_{0}^{1} \int_{0}^{1}\left[\frac{\left(R_{12} f\right)(u, v)}{(\log e / u)^{a}(\log e / v)^{b}}\right]^{q} \frac{d u}{u \log e / u} \frac{d v}{v \log e / v}\right)^{1 / q},
\end{aligned}
$$

which proves the claim. 
Corollary 2.10. Let $1<q<\infty$. Then

$$
\begin{aligned}
& \sup _{0<s<1} \sup _{0<t<1} \frac{\left(R_{12} f\right)(s, t)}{[(\log e / s)(\log e / t)]^{1 / d^{\prime}}} \\
& \leq c\left(\int_{0}^{1} \int_{0}^{1}\left[\frac{\left(R_{12} f\right)(s, t)}{(\log e / s)(\log e / t)}\right]^{d} \frac{d s}{s} \frac{d t}{t}\right)^{1 / d} \\
& \leq c\left(\int_{0}^{1} \int_{0}^{1}\left[\frac{\left(R_{12} f\right)(s, t)}{(\log e / s)(\log e / t)}\right]^{r} \frac{d s}{s} \frac{d t}{t}\right)^{1 / r}
\end{aligned}
$$

for every $1<r<d<\infty, 1 / d+1 / d^{\prime}=1$.

Proof. Let $1<d<\infty$. Then the first inequality in (2.21) follows immediately from Proposition 2.9 on putting $a=b=1 / d^{\prime}$.

If $1<r<d$, then discretizing the middle term in (2.21) we get

$$
\begin{aligned}
&\left(\int_{0}^{1} \int_{0}^{1}\left[\frac{\left(R_{12} f\right)(s, t)}{(\log e / s)(\log e / t)}\right]^{d}\right. \frac{d s}{s} \\
&\left.\frac{d t}{t}\right)^{1 / d} \sim\left(\sum_{k=1}^{\infty} \sum_{l=1}^{\infty}\left[\frac{\left(R_{12} f\right)\left(2^{-k}, 2^{l}\right)}{k l}\right]^{d}\right)^{1 / d} \\
& \leq\left(\sum_{k=1}^{\infty}\left[\frac{\left(R_{12} f\right)\left(2^{-k}, 2^{l}\right)}{k l}\right]^{r}\right)^{1 / r} \\
& \leq c\left(\int_{0}^{1} \int_{0}^{1}\left[\frac{\left(R_{12} f\right)(s, t)}{(\log e / s)(\log e / t)}\right]^{r} \frac{d s}{s} \frac{d t}{t}\right)^{1 / r} .
\end{aligned}
$$

REMARK 2.11. If we use the Orlicz-Lorentz type notations mentioned earlier, the inequalities in (2.21) express imbeddings

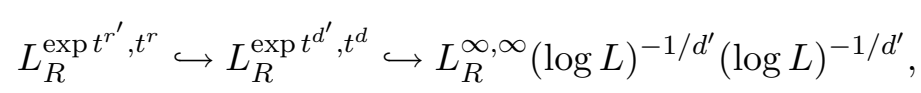

which are nothing else than Orlicz type generalizations of monotonicity properties of usual Lorentz spaces with respect to parameters. Here, one has to replace inequalities for parameters of Lorentz spaces by the usual ordering of Young functions: $\Psi_{1} \prec \Psi_{2}$ if there exists $c>0$ such that $\Psi_{1}(t) \leq \Psi_{2}(c t)$ for large $t$. A similar interpretation, giving a more transparent idea of behaviour of the multivariate exponential spaces, can also be given for other inequalities above.

2.3. Spaces with dominating mixed smoothness. Let us recall the definitions of the spaces we shall work with. Let $\psi$ be a real-valued infinitely differentiable function such that $0 \leq \psi(x) \leq 1, \psi(x)=1$ if $|x| \leq 1$, and $\psi(x)=0$ if $|x| \geq 2$. Put 


$$
\begin{aligned}
& \varphi_{0}(x)=\psi(x) \\
& \varphi_{1}(x)=\varphi_{0}(x / 2)-\varphi_{0}(x), \\
& \varphi_{j}(x)=\varphi_{1}\left(2^{-j+1} x\right), \quad j=2,3, \ldots
\end{aligned}
$$

Plainly,

$$
\sum_{j=0}^{\infty} \varphi_{j}(x)=1, \quad x \in \mathbb{R}^{N} .
$$

The system of functions $\left\{\varphi_{j}\right\}$ is a smooth dyadic resolution of unity.

Now we give the definitions of the spaces with dominating mixed derivatives. We shall restrict ourselves to the special splitting $\mathbb{R}^{2 N}=\mathbb{R}^{N} \times \mathbb{R}^{N}$.

Let $\left\{\varphi_{j}\right\}_{j=0}^{\infty}$ be a smooth dyadic resolution of unity in $\mathbb{R}^{N}$. For $f \in$ $\mathcal{S}^{\prime}\left(\mathbb{R}^{2 N}\right)$ we put

$$
f_{j, k}(x, y):=\left[\mathcal{F}^{-1} \varphi_{j}(\xi) \varphi_{k}(\eta) \mathcal{F} f\right](x, y) \quad(j, k=0,1, \ldots) .
$$

DEFINITION 2.12.

(i) Let $0<p, q \leq \infty, r \in \mathbb{R}^{1}$. Then

$$
\begin{aligned}
S_{p q}^{r} B\left(\mathbb{R}^{N} \times \mathbb{R}^{N}\right)=\left\{f \in \mathcal{S}^{\prime}\left(\mathbb{R}^{2 N}\right)\right. & :\left\|f \mid S_{p q}^{r} B\left(\mathbb{R}^{N} \times \mathbb{R}^{N}\right)\right\| \\
& \left.=\left\|2^{r j+r k} f_{j, k}(x, y)\left|L_{p}\right| \ell_{q}\right\|<\infty\right\} .
\end{aligned}
$$

(ii) Let additionally $0<p<\infty$. Then

$$
\begin{aligned}
S_{p q}^{r} F\left(\mathbb{R}^{N} \times \mathbb{R}^{N}\right)=\left\{f \in \mathcal{S}^{\prime}\left(\mathbb{R}^{2 N}\right)\right. & :\left\|f \mid S_{p q}^{r} F\left(\mathbb{R}^{N} \times \mathbb{R}^{N}\right)\right\| \\
& \left.=\left\|2^{r j+r k} f_{j, k}(x, y)\left|\ell_{q}\right| L_{p}\right\|<\infty\right\} .
\end{aligned}
$$

Of course the mixed norms in the definition of $S_{p q}^{r} B\left(\mathbb{R}^{N} \times \mathbb{R}^{N}\right)$ should be understood in the sense

$$
\left\|2^{r j+r k} f_{j, k}(x, y)\left|L_{p}\left(\mathbb{R}^{N} \times \mathbb{R}^{N}\right)\right| \ell_{q}\left(\mathbb{N}_{0} \times \mathbb{N}_{0}\right)\right\|,
$$

and similarly in (ii).

For the definition of the usual Besov and Lizorkin-Triebel spaces $B_{p q}^{r}\left(\mathbb{R}^{M}\right)$ and $F_{p q}^{r}\left(\mathbb{R}^{M}\right)$ we refer to [Tri1].

REMARK 2.13. The spaces defined above are special cases of those introduced and studied in [ScT, Chapter 2] and one can find further references there. Up to equivalent (quasi)norms these spaces do not depend on the choice of the dyadic resolutions of unity. In [ScT], further equivalent characterizations are given, in particular in terms of differences and derivatives.

REMARK 2.14. We recall important properties of the Sobolev spaces with dominating mixed smoothness (see [ScT, Chapter 2]). Let $1<p<\infty$. Then 


$$
\begin{aligned}
& S_{p 2}^{r} F\left(\mathbb{R}^{N} \times \mathbb{R}^{N}\right)=S_{p}^{r} H\left(\mathbb{R}^{N} \times \mathbb{R}^{N}\right) \\
& :=\left\{f \in \mathcal{S}^{\prime}\left(\mathbb{R}^{2 N}\right):\left\|f \mid S_{p}^{r} H\left(\mathbb{R}^{N} \times \mathbb{R}^{N}\right)\right\|\right. \\
& \left.\quad=\left\|\mathcal{F}^{-1}\left[\left(1+|\xi|^{2}\right)^{r / 2}\left(1+|\eta|^{2}\right)^{r / 2} \mathcal{F} f\right] \mid L_{p}\left(\mathbb{R}^{n} \times \mathbb{R}^{N}\right)\right\|<\infty\right\} .
\end{aligned}
$$

If additionally $r=0,1,2, \ldots$, then $S_{p}^{r} H\left(\mathbb{R}^{N} \times \mathbb{R}^{N}\right)=S_{p}^{r} W\left(\mathbb{R}^{2 N}\right)$, where

$$
S_{p}^{r} W\left(\mathbb{R}^{2 N}\right):=\left\{f \in L_{p}\left(\mathbb{R}^{2 N}\right): D^{\left(\alpha^{1}, \alpha^{2}\right)} f \in L_{p},\left|\alpha^{j}\right| \leq r(j=1,2)\right\},
$$

and where $D^{\left(\alpha^{1}, \alpha^{2}\right)}$ stands for $D_{x}^{\alpha^{1}} D_{y}^{\alpha^{2}}$, with equivalence of the corresponding norms. Observe also that

$$
\begin{aligned}
\| f \mid S_{p}^{r} W\left(\mathbb{R}^{2 N}\right) & \sim\left\|f\left|L_{p}\left(\mathbb{R}^{2 N}\right)\left\|+\sum_{j=1}^{N}\right\| \frac{\partial^{r} f}{\partial x_{j}^{r}}\right| L_{p}\left(\mathbb{R}^{2 N}\right)\right\| \\
& +\sum_{j=1}^{N}\left\|\frac{\partial^{r} f}{\partial y_{j}^{r}}\left|L_{p}\left(\mathbb{R}^{2 N}\right)\left\|+\sum_{j, k=1}^{N}\right\| \frac{\partial^{2 r} f}{\partial x_{j}^{r} \partial y_{k}^{r}}\right| L_{p}\left(\mathbb{R}^{2 N}\right)\right\|,
\end{aligned}
$$

which is a very illustrative justification for the name of the spaces: there are no "pure" derivatives of order greater than $r$, the mixed derivatives of order $2 r$ "dominate".

Note that $S_{p}^{0} H\left(\mathbb{R}^{N} \times \mathbb{R}^{N}\right)=L_{p}\left(\mathbb{R}^{2 N}\right)$.

REMARK 2.15. The following sharp imbeddings hold (see [ScT] and [ScSi] for details):

$$
S_{p_{0} q_{0}}^{r_{0}} B\left(\mathbb{R}^{N} \times \mathbb{R}^{N}\right) \hookrightarrow S_{p q}^{r} B\left(\mathbb{R}^{N} \times \mathbb{R}^{N}\right)
$$

if

$$
0<p_{0}<p \leq \infty, \quad 0<q_{0} \leq q \leq \infty, \quad r<r_{0}, \quad r_{0}-\frac{N}{p_{0}}=r-\frac{N}{p},
$$

and

$$
S_{p_{0} q_{0}}^{r_{0}} F\left(\mathbb{R}^{N} \times \mathbb{R}^{N}\right) \hookrightarrow S_{p p_{0}}^{r} B\left(\mathbb{R}^{N} \times \mathbb{R}^{N}\right)
$$

if

$$
0<p_{0}<p<\infty, \quad 0<q_{0} \leq \infty, \quad r<r_{0}, \quad r_{0}-\frac{N}{p_{0}}=r-\frac{N}{p}
$$

3. The main theorem. We shall investigate the imbeddings of the spaces $S_{p q}^{N / p} B\left(\mathbb{R}^{N} \times \mathbb{R}^{N}\right)$ for $0<p<\infty, 1<q \leq \infty$, as well as of $S_{p q}^{N / p} F\left(\mathbb{R}^{N} \times \mathbb{R}^{N}\right)$ for $1<p<\infty, 0<q \leq \infty$, into multivariate LorentzOrlicz spaces. Our approach will be based on atomic characterization (see [Tri2], [H]), which permits a reduction of integral inequalities to corresponding problems in sequence spaces, which are easier to handle. 
Our main result reads as follows:

Theorem 3.1. Let $0<p<\infty$ and $1<q \leq \infty$. Then

$$
\int_{0}^{1} \int_{0}^{1}\left[\frac{\left(A_{12} f\right)(s, t)}{(\log e / s)(\log e / t)}\right]^{q} \frac{d s}{s} \frac{d t}{t} \leq c\left\|f \mid S_{p q}^{N / p} B\left(\mathbb{R}^{N} \times \mathbb{R}^{N}\right)\right\|^{q}
$$

and

$$
\sup _{0<s \leq 1} \sup _{0<t \leq 1} \frac{\left(A_{12} f\right)(s, t)}{[(\log e / s)(\log e / t)]^{1 / q^{\prime}}} \leq c\left\|f \mid S_{p q}^{N / p} B\left(\mathbb{R}^{N} \times \mathbb{R}^{N}\right)\right\|
$$

for all $f \in S_{p q}^{N / p} B\left(\mathbb{R}^{N} \times \mathbb{R}^{N}\right.$ ) (with an appropriate modification if $q=\infty$ ). Analogous inequalities hold for $A_{21} f$.

Corollary 3.2. Let $1<p<\infty$ and $0<q \leq \infty$. Then

$$
\int_{0}^{1} \int_{0}^{1}\left(\frac{\left(A_{12} f\right)(s, t)}{(\log e / s)(\log e / t)}\right)^{p} \frac{d s}{s} \frac{d t}{t} \leq\left\|f \mid S_{p q}^{N / p} F\left(\mathbb{R}^{N} \times \mathbb{R}^{N}\right)\right\|^{p} .
$$

Proof. Using the imbeddings in Remark 2.15 we get

$$
S_{p q}^{N / p} F\left(\mathbb{R}^{N} \times \mathbb{R}^{N}\right) \hookrightarrow S_{u p}^{N / u} B\left(\mathbb{R}^{N} \times \mathbb{R}^{N}\right)
$$

if $1<p<u<\infty$. Now (3.3) is a consequence of (3.1).

REMARK 3.3. If we take into account the equivalences (2.22) and (2.23), Corollary 3.2 also applies to the spaces $S_{p}^{N / p} H\left(\mathbb{R}^{N} \times \mathbb{R}^{N}\right)$ and $S_{p}^{N / p} W\left(\mathbb{R}^{2 N}\right)$.

A natural question arises about the case $0<q \leq 1$ in Theorem 3.1 and $0<p \leq 1$ in Corollary 3.2. Invoking imbedding theorems in [ScT] and the sharp imbeddings from Remark 2.15 one can infer that in both cases the functions in these spaces are uniformly continuous and bounded in $\mathbb{R}^{2 N}$.

REMARK 3.4. The Sobolev imbeddings in the subcritical case have been recently refined in $[\mathrm{K}]$ with the use of multivariate rearrangements. For simplicity, let us restrict ourselves to the case $N=2$ and assume that $r=1$ and $1<p<2$. Then

$$
W_{p}^{1}\left(\mathbb{R}^{2}\right)=H_{p}^{1}\left(\mathbb{R}^{2}\right) \hookrightarrow L_{R}^{p^{*}, p}\left(\mathbb{R}^{2}\right),
$$

where $H_{p}^{1}\left(\mathbb{R}^{2}\right)$ is the space of Bessel potentials and

$$
p^{*}=\frac{2 p}{2-p}
$$

(see (2.7) for the definition of the target space).

An example of critical imbeddings of the "standard" Besov spaces in $\mathbb{R}^{2 N}$ and of $S_{p q}^{N / p} B\left(\mathbb{R}^{N} \times \mathbb{R}^{N}\right)$, throwing some more light on the structure of the spaces with dominating mixed derivatives, can be found after the proof of the main theorem. 
Note for completeness that the multivariate rearrangements have also been used recently to strengthen subcritical imbeddings of anisotropic Lipschitz spaces in $[\mathrm{P}]$.

Proof of Theorem 3.1. Step 1. Since $A_{12} f$ is non-increasing with respect to $s$ and $t$ we can discretize the integral on the left hand side of (3.1). For any $D \geq 2^{N}$ we obtain

$$
\begin{gathered}
\int_{00}^{1} \int_{0}^{1}\left[\frac{\left(A_{12} f\right)(s, t)}{(\log e / s)(\log e / t)}\right]^{q} \frac{d s}{s} \frac{d t}{t} \\
\leq \sum_{k=1}^{\infty} \int_{2^{-(k+1) N} D}^{2^{-k N} D} \sum_{l=k_{0}}^{\infty} \int_{2^{-(l+1) N} D}^{2^{-l N} D}\left[\frac{\left(A_{12} f\right)(s, t)}{(\log e / s)(\log e / t)}\right]^{q} \frac{d s}{s} \frac{d t}{t} \\
\leq c \sum_{k=1}^{\infty} \sum_{l=1}^{\infty}\left[\frac{\left(A_{12} f\right)\left(2^{-k N} D, 2^{-l N} D\right)}{k l}\right]^{q} .
\end{gathered}
$$

Note that the constant $c$ may depend on the choice of $D$.

Step 2. By the first imbedding in Remark 2.15 we can suppose that $p>1$. Recall (see $[\mathrm{R}]$ and $[\mathrm{V}]$ ) that $f \in S_{p q}^{N / p} B\left(\mathbb{R}^{N} \times \mathbb{R}^{N}\right.$ ) if and only if there is a decomposition

$$
f=\sum_{i=0}^{\infty} \sum_{j=0}^{\infty} \sum_{m \in \mathbb{Z}^{N}} \sum_{n \in \mathbb{Z}^{N}} b_{i, j}^{m, n} a_{i, j}^{m, n}(x, y),
$$

where $a_{i, j}^{m, n}(x, y)$ are atoms, that is,

- $a_{i, j}^{m, n} \in C_{0}^{\infty}\left(\mathbb{R}^{N} \times \mathbb{R}^{N}\right)$,

- $\operatorname{supp} a_{i, j}^{m, n} \subset d Q_{i}(m) \times d Q_{j}(n)$ with some $d>1$, where $Q_{i}(m)=\{y$ : $\left.2^{-i} m_{r} \leq y_{r}<2^{-i}\left(m_{r}+1\right), r=1, \ldots, N\right\}$,

- $\left|D^{(\alpha, \beta)} a_{i, j}^{m, n}(x, y)\right| \leq 2^{i|\alpha|+j|\beta|}$,

and

$$
\|b\|_{p, q}^{q}:=\sum_{(i, j) \in \mathbb{N}_{0} \times \mathbb{N}_{0}}\left(\sum_{(m, n) \in \mathbb{Z}^{N} \times \mathbb{Z}^{N}}\left|b_{i, j}^{m, n}\right|^{p}\right)^{q / p}<\infty .
$$

Moreover,

$$
\inf _{b}\|b\|_{p, q} \sim\left\|f \mid S_{p q}^{N / p} B\left(\mathbb{R}^{N} \times \mathbb{R}^{N}\right)\right\| .
$$

Step 3. Let $f$ be decomposed as in (3.5). We wish to estimate the right hand side of (3.1) by (3.6) multiplied by a constant independent of the choice of the coefficients $b_{i, j}^{m, n}$ and atoms $a_{i, j}^{m, n}(x, y)$. Put

$$
f_{i, j}(x, y)=\sum_{m \in \mathbb{Z}^{N}} \sum_{n \in \mathbb{Z}^{N}} b_{i, j}^{m, n} a_{i, j}^{m, n}(x, y), \quad(i, j) \in \mathbb{N}_{0} \times \mathbb{N}_{0} .
$$


By (3.5), (3.7), and the subadditivity of $A_{12}$ we have

$$
\begin{aligned}
& \sum_{k=1}^{\infty} \sum_{l=1}^{\infty}\left[\frac{\left(A_{12} f\right)\left(2^{-k N} D, 2^{-l N} D\right)}{k l}\right]^{q} \\
& \leq \sum_{k=1}^{\infty} \sum_{l=1}^{\infty}\left[\frac{1}{k l} \sum_{i=0}^{\infty} \sum_{j=0}^{\infty}\left(A_{12} f_{i, j}\right)\left(2^{-k N} D, 2^{-l N} D\right)\right]^{q}
\end{aligned}
$$

Denote by $\mathrm{RH}(f)$ the expression on the right hand side of (3.8). We estimate $\mathrm{RH}(f)$ by four terms as follows:

$$
\begin{aligned}
R H(f) \leq & \sum_{k=1}^{\infty} \sum_{l=1}^{\infty}\left[\frac{1}{k l} \sum_{i=0}^{k} \sum_{j=0}^{l}\left(A_{12} f_{i, j}\right)\left(2^{-k N} D, 2^{-l N} D\right)\right]^{q} \\
& +\sum_{k=1}^{\infty} \sum_{l=1}^{\infty}\left[\frac{1}{k l} \sum_{i=k+1}^{\infty} \sum_{j=l+1}^{\infty}\left(A_{12} f_{i, j}\right)\left(2^{-k N} D, 2^{-l N} D\right)\right]^{q} \\
& +\sum_{k=1}^{\infty} \sum_{l=1}^{\infty}\left[\frac{1}{k l} \sum_{i=0}^{k} \sum_{j=l+1}^{\infty}\left(A_{12} f_{i, j}\right)\left(2^{-k N} D, 2^{-l N} D\right)\right]^{q} \\
& +\sum_{k=1}^{\infty} \sum_{l=1}^{\infty}\left[\frac{1}{k l} \sum_{i=k+1}^{\infty} \sum_{j=0}^{l}\left(A_{12} f_{i, j}\right)\left(2^{-k N} D, 2^{-l N} D\right)\right]^{q} \\
= & \sum_{1}+\sum_{2}+\sum_{3}+\sum_{4} .
\end{aligned}
$$

Step 4. We claim that there exist constants $c>0$ and $D \geq 2^{N}$ such that

$$
\left(A_{12} f_{i, j}\right)(s, t) \leq \frac{c}{\mu \nu} \sum_{\alpha=1}^{\mu} \sum_{\beta=1}^{\nu}\left(R_{12} b_{i, j}\right)(\alpha, \beta)=b_{i, j}^{* *}(\mu, \nu)
$$

provided

$$
(s, t) \in\left(2^{-i N} D(\mu-1), 2^{-i N} D \mu\right] \times\left(2^{-j N} D(\nu-1), 2^{-i N} D \nu\right], \mu, \nu \in \mathbb{N} .
$$

To show this recall that

$$
f_{i, j}(x, y)=\sum_{m \in \mathbb{Z}^{N}} \sum_{n \in \mathbb{Z}^{N}} b_{i, j}^{m, n} a_{i, j}^{m, n}(x, y)
$$

and since the atoms are bounded from above by 1 we have

$$
\left|f_{i, j}(x, y)\right| \leq \sum_{\text {fin }} \sum_{m \in \mathbb{Z}^{N}} \sum_{n \in \mathbb{Z}^{N}}\left|b_{i, j}^{m, n}\right| \chi_{i, j}^{m+\widetilde{m}, n+\widetilde{n}}(x, y),
$$

where $\chi_{i, j}^{m+\widetilde{m}, n+\widetilde{n}}$ is the characteristic function of the cube $Q_{i, j}(m+\widetilde{m}$, $n+\widetilde{n})=Q_{i}(m+\widetilde{m}) \times Q_{j}(n+\widetilde{n})$ and by the properties of atoms (compact supports) the first sum is taken over a finite index set, whose cardinality is 
independent of $i$ and $j$. Hence there exists a constant $c_{1} \in(0,1 / 2]$ such that

$$
\left(R_{12} f_{i, j}\right)(s, t) \leq \sum_{\text {fin }} R_{12}\left(\sum_{m \in \mathbb{Z}^{N}} \sum_{n \in \mathbb{Z}^{N}}\left|b_{i, j}^{m, n}\right| \chi_{i, j}^{m+\widetilde{m}, n+\widetilde{n}}\right)\left(c_{1} s, c_{1} t\right)
$$

and this is

$$
\begin{aligned}
& \leq c R_{12}\left(\sum_{m \in \mathbb{Z}^{N}} \sum_{n \in \mathbb{Z}^{N}}\left|b_{i, j}^{m, n}\right| \chi_{i, j}^{m, n}\right)\left(c_{1} s, c_{1} t\right) \\
& =c \sum_{\mu=1}^{\infty} \sum_{\nu=1}^{\infty}\left(R_{12} b_{i, j}\right)(\mu, \nu)\left(\chi_{i}^{\mu}\right)(s)\left(\chi_{j}^{\nu}\right)(t),
\end{aligned}
$$

where $\chi_{i}^{\mu}$ stands for the (one-dimensional) characteristic function of the interval $\left[2^{-i N} c_{1}^{-N}(\mu-1), 2^{-i N} c_{1}^{-N} \mu\right)$. Therefore if (3.11) holds with $D=$ $c_{1}^{-N} \geq 2^{N}$, we have, invoking (2.4),

$$
\begin{aligned}
\left(A_{12} f_{i, j}\right)(s, t) & \leq \frac{1}{s t} \int_{0}^{t s}\left(R_{12} f_{i, j}\right)(u, v) d u d v \\
& \leq c 2^{i N+j N} \frac{1}{\mu \nu} \sum_{\alpha=1}^{\mu} \sum_{\beta=1}^{\nu}\left(R_{12} b_{i, j}\right)(\alpha, \beta) \int_{0}^{t} \chi_{i}^{\alpha}(u) d u \int_{0}^{s} \chi_{j}^{\beta}(v) d v .
\end{aligned}
$$

Since the last two integrals are $\leq 2^{-i N} D$ and $\leq 2^{-j N} D$, resp., this is

$$
\leq \frac{c}{\mu \nu} \sum_{\alpha=1}^{\mu} \sum_{\beta=1}^{\nu}\left(R_{12} b_{i, j}\right)(\alpha, \beta) .
$$

Summarizing the above estimates we have (3.10) for all $(s, t)$ satisfying (3.11).

Step 5. We estimate $\sum_{1}$. Since $i \leq k$ and $j \leq l$ we have

$$
\left(2^{-k N} D, 2^{-l N} D\right) \in\left(0,2^{-i N} D\right] \times\left(0,2^{-j N} D\right] .
$$

The estimate (3.10) with $\mu=\nu=1$ yields

$$
\left(A_{12} f_{i, j}\right)\left(2^{-k N} D, 2^{-l N} D\right) \leq c\left(R_{12} b_{i, j}\right)(1,1) .
$$

Hence by the maximal inequality from Lemma 2.1 we obtain, for any $1<$ $q \leq \infty$ and $1<p<\infty$,

$$
\begin{aligned}
\sum_{1} & \leq c \sum_{k=1}^{\infty} \sum_{l=1}^{\infty}\left[\frac{1}{k l} \sum_{i=0}^{k} \sum_{j=0}^{l}\left(R_{12} b_{i, j}\right)(1,1)\right]^{q} \leq c \sum_{i=0}^{\infty} \sum_{j=0}^{\infty}\left[\left(R_{12} b_{i, j}\right)(1,1)\right]^{q} \\
& \leq c \sum_{i=0}^{\infty} \sum_{j=0}^{\infty}\left[\max _{m, n}\left|b_{i, j}^{m, n}\right|\right]^{q} \leq c \sum_{i=0}^{\infty} \sum_{j=0}^{\infty}\left(\sum_{m \in \mathbb{Z}^{N}} \sum_{n \in \mathbb{Z}^{N}}\left|b_{i, j}^{m, n}\right|^{p}\right)^{q / p}=c\|b\|_{p, q}^{q} .
\end{aligned}
$$


Step 6. We shall estimate the term $\sum_{2}$. Fix $i \in\{k+1, \ldots\}$ and $j \in$ $\{l+1, \ldots\}$. We have

$$
2^{-k N} D=2^{-i N} D 2^{(i-k) N}, \quad 2^{-l N} D=2^{-j N} D 2^{(j-l) N} .
$$

The estimate (3.10) with $\mu=2^{(i-k) N}$ and $\nu=2^{(j-l) N}$ implies

$$
\begin{aligned}
& \left(A_{12} f_{i, j}\right)\left(2^{-k N} D, 2^{-l N} D\right) \\
& \leq c \frac{1}{2^{(i-k) N}} \frac{1}{2^{(j-l) N}} \sum_{\alpha=1}^{2^{(i-k) N}} \sum_{\beta=1}^{2^{(j-l) N}}\left(R_{12} b_{i, j}\right)(\alpha, \beta) .
\end{aligned}
$$

We have

$$
\left[b_{i, j}^{* *}\left(2^{\kappa N}, 2^{\lambda N}\right)\right]^{p} \leq\left[b_{i, j}^{* *}(\varrho, \sigma)\right]^{p}
$$

for $\kappa, \lambda=1,2, \ldots$, and

$$
2^{(\kappa-1) N}<\varrho \leq 2^{\kappa N}, \quad 2^{(\lambda-1) N}<\sigma \leq 2^{\lambda N} .
$$

Summing up (3.13) over such $\varrho$ and $\sigma$ we get (the number of terms on the left is $\left.2^{(\kappa-1) N+(\lambda-1) N}\left(2^{N}-1\right)^{2}\right)$

$$
2^{(\kappa-1) N+(\lambda-1) N}\left[b_{i, j}^{* *}\left(2^{\kappa N}, 2^{\lambda N}\right)\right]^{p} \leq \sum_{\varrho=2^{(\kappa-1) N}+1}^{2^{\kappa N}} \sum_{\sigma=2^{(\lambda-1) N}+1}^{2^{\lambda N}}\left[b_{i, j}^{* *}(\varrho, \sigma)\right]^{p} .
$$

It follows that

$$
\left[b_{i, j}^{* *}\left(2^{\kappa N}, 2^{\lambda N}\right)\right]^{p} \leq c 2^{-(\kappa+\lambda) N} \sum_{\varrho=1}^{\infty} \sum_{\sigma=1}^{\infty}\left[b_{i, j}^{* *}(\varrho, \sigma)\right]^{p} .
$$

After applying the maximal inequality for sequences this is

$$
\begin{aligned}
& \leq c 2^{-(\kappa+\lambda) N} \sum_{\alpha=1}^{\infty} \sum_{\beta=1}^{\infty}\left[\left(R_{12}\right)(\alpha, \beta)\right]^{p} \\
& =c 2^{-(\kappa+\lambda) N} \sum_{m \in \mathbb{Z}^{N}} \sum_{n \in \mathbb{Z}^{N}}\left|b_{i, j}^{m, n}\right|^{p} \\
& =c 2^{-(\kappa+\lambda) N}\left\|b_{i, j} \mid \ell_{p}\right\|^{p} .
\end{aligned}
$$

Putting $\kappa=i-k$ and $\lambda=j-l$ we see from (3.14) and (3.12) that

$$
\left(A_{12} f_{i, j}\right)\left(2^{-k N} D, 2^{-l N} D\right) \leq c 2^{-(i-k) N / p-(j-l) N / p}\left\|b_{i, j} \mid \ell_{p}\right\| .
$$

This implies

$$
\sum_{2} \leq c \sum_{k=1}^{\infty} \sum_{l=1}^{\infty}\left[\frac{1}{k^{q} l^{q}} \sum_{i=k+1}^{\infty} \sum_{j=l+1}^{\infty} 2^{-(i-k) N / p-(j-l) N / p}\left\|b_{i, j} \mid \ell_{p}\right\|\right]^{q} .
$$

By Hölder's inequality it follows for $q>1$ that

$$
\sum_{2} \leq c\|b\|_{p, q}^{q} \text {. }
$$


Step 7. We estimate the term $\sum_{3}$. Fix $i=0,1, \ldots, k$ and $j=l+1$, $l+2, \ldots$ Then $2^{-k N} D \in\left(0,2^{-i N} D\right]$ and $2^{-l N} D=2^{-j N} D 2^{(j-l) N}$. The estimate (3.10) from Step 4 with $\mu=1$ and $\nu=2^{(j-l) N}$ yields

$$
\left(A_{12} f_{i, j}\right)\left(2^{-k N} D, 2^{-l N} D\right) \leq \frac{c}{2^{(j-l) N}} \sum_{\beta=1}^{2^{(j-l) N}}\left(R_{12} b_{i, j}\right)(1, \beta) .
$$

Arguing as before in Step 6 we get, for $p>1$ and $\lambda=1,2, \ldots$,

$$
\begin{aligned}
{\left[\frac{1}{2^{\lambda N}} \sum_{\beta=1}^{2^{\lambda N}}\left(R_{12} b_{i, j}\right)(1, \beta)\right]^{p} } & =\left[\left(A_{2} \circ R_{1} b_{i, j}\right)\left(1,2^{\lambda N}\right)\right]^{p} \\
& \leq c 2^{-\kappa N} \sum_{\sigma=1}^{\infty}\left[\left(A_{2} \circ R_{1} b_{i, j}\right)(1, \sigma)\right]^{p}
\end{aligned}
$$

and by the maximal inequality this is

$$
\leq c 2^{-\lambda N} \sum_{\beta=1}^{\infty}\left[\left(R_{2} \circ R_{1} b_{i, j}\right)(1, \beta)\right]^{p} \leq c 2^{-\lambda N} \sum_{n \in \mathbb{Z}^{N}}\left|\left(R_{1} b_{i, j}\right)(1, n)\right|^{p} .
$$

Invoking (3.15) we obtain

$$
\begin{aligned}
& \left(A_{12} f_{i, j}\right)\left(2^{-k N} D, 2^{-l N} D\right) \leq c 2^{-(j-l) N / p}\left(\sum_{m \in \mathbb{Z}^{N}}\left|\left(R_{1} b_{i, j}\right)(1, n)\right|^{p}\right)^{1 / p} \\
& \leq c 2^{-(j-l) N / p}\left(\sum_{m \in \mathbb{Z}^{N}} \sum_{n \in \mathbb{Z}^{N}}\left|b_{i, j}^{m, n}\right|^{p}\right)^{1 / p}=c 2^{-(j-l) N / p}\left\|b_{i, j} \mid \ell_{p}\right\| .
\end{aligned}
$$

Inserting (3.15) and (3.16) into $\sum_{3}$ we get

$$
\sum_{3} \leq c \sum_{k=1}^{\infty} \sum_{l=1}^{\infty}\left(\frac{1}{k l} \sum_{i=0}^{k} \sum_{j=l+1}^{\infty} 2^{-(j-l) N / p}\left\|b_{i, j} \mid \ell_{p}\right\|\right)^{q} .
$$

If we set $c_{i, l}=\sum_{j=l+1}^{\infty} 2^{-(j-l) N / p}\left\|b_{i, j} \mid \ell_{p}\right\|$, this becomes

$$
=c \sum_{l=1}^{\infty} \frac{1}{l^{q}} \sum_{k=1}^{\infty}\left(\frac{1}{k} \sum_{i=0}^{k} c_{i, l}\right)^{q}
$$

and by the maximal inequality this is

$$
\leq c \sum_{l=1}^{\infty} \frac{1}{l^{q}} \sum_{i=0}^{\infty} c_{i, l}^{q}=c \sum_{i=0}^{\infty} \sum_{l=1}^{\infty} \frac{1}{l^{q}}\left(\sum_{j=l+1}^{\infty} 2^{-(j-l) N / p}\left\|b_{i, j} \mid \ell_{p}\right\|\right)^{q} .
$$


By Hölder's inequality the last expression above is

$$
\begin{aligned}
& \leq c \sum_{i=0}^{\infty} \sum_{l=1}^{\infty} \frac{1}{l^{q}}\left(\sum_{j=l+1}^{\infty} 2^{-(j-l) N q^{\prime} / p}\right)^{q / q^{\prime}} \sum_{j=0}^{\infty}\left\|b_{i, j} \mid \ell_{p}\right\|^{q} \\
& \leq c\left(\sum_{l=1}^{\infty} \frac{1}{l^{q}}\right) \sum_{i=0}^{\infty} \sum_{j=0}^{\infty}\left\|b_{i, j} \mid \ell_{p}\right\|^{q}=c\|b\|_{p, q}^{q} .
\end{aligned}
$$

Step 8. It remains to establish an estimate for $\sum_{4}$. Fix $i=k+1, \ldots$ and $j=0, \ldots, l$. Then $2^{-k N} D=2^{-i N} D 2^{(i-k) N}$ and $2^{-l N} D \in\left(0,2^{-j N} D\right]$. Employing (3.10) with $\mu=2^{(i-k) N}$ and $\nu=1$ and arguing as in Step 6, we can write

$$
\begin{aligned}
& \left(A_{12} f_{i, j}\right)\left(2^{-k N} D, 2^{-l N} D\right) \leq \frac{c}{2^{(i-k) N}} \sum_{\alpha=1}^{2^{(i-k) N}}\left(R_{12} b_{i, j}\right)(\alpha, 1) \\
& =c\left(b_{i, j}\right)^{* *}\left(2^{(i-k) N}, 1\right) \leq c 2^{-(i-k) N / p}\left(\sum_{\varrho=1}^{\infty}\left[\left(b_{i, j}\right)^{* *}(\varrho, 1)\right]^{p}\right)^{1 / p}
\end{aligned}
$$

which, after application of the maximal inequality, is

$$
\leq c 2^{-(i-k) N / p}\left(\sum_{\alpha=1}^{\infty} \sum_{\beta=1}^{\infty}\left|\left(R_{12} b_{i, j}\right)(\alpha, \beta)\right|^{p}\right)^{1 / p}=c 2^{-(i-k) N / p}\left\|b_{i, j} \mid \ell_{p}\right\|
$$

Inserting (3.17) into $\sum_{4}$ gives

$$
\begin{aligned}
\sum_{4} & \leq c \sum_{k=0}^{\infty} \sum_{l=0}^{\infty}\left(\frac{1}{k l} \sum_{i=k+1}^{\infty} \sum_{j=0}^{l} 2^{-(i-k) N / p}\left\|b_{i, j} \mid \ell_{p}\right\|\right)^{q} \\
& =c \sum_{k=0}^{\infty} \frac{1}{k^{q}} \sum_{l=0}^{\infty}\left[\frac{1}{l} \sum_{j=0}^{l}\left(\sum_{i=k+1}^{\infty} 2^{-(i-k) N / p}\left\|b_{i, j} \mid \ell_{p}\right\|\right)\right]^{q} .
\end{aligned}
$$

Define

$$
d_{k, j}=\sum_{i=k+1}^{\infty} 2^{-(i-k) N / p}\left\|b_{i, j} \mid \ell_{p}\right\| .
$$

Then by Hölder's inequality,

$$
d_{k, j} \leq c\left(\sum_{i=0}^{\infty}\left\|b_{i, j} \mid \ell_{p}\right\|^{q}\right)^{1 / q}=: e_{j}
$$

independently of $k$. Applying once more the maximal inequality we arrive at

$$
\sum_{4} \leq \sum_{l=0}^{\infty}\left(\frac{1}{l} \sum_{j=0}^{l} e_{j}\right)^{q} \leq c \sum_{j=0}^{\infty} e_{j}^{q}=c \sum_{j=0}^{\infty} \sum_{i=0}^{\infty}\left\|b_{i, j} \mid \ell_{p}\right\|^{q}=c\|b\|_{p, q}^{q} .
$$


Finally, the estimate (3.2) follows directly from (3.1) and Corollary 2.10. The theorem is proved.

4. Sharpness and envelopes. In this section we discuss the sharpness of estimates (3.1) and (3.3). First we observe that

$$
\left\|f_{1} \otimes f_{2}\left|S_{p q}^{r} B\left(\mathbb{R}^{N} \times \mathbb{R}^{N}\right)\|=\| f_{1}\right| B_{p q}^{r}\left(\mathbb{R}^{N}\right)\right\|\left\|f_{2} \mid B_{p q}^{r}\left(\mathbb{R}^{N}\right)\right\|
$$

and

$$
\left\|g_{1} \otimes g_{2}\left|S_{p q}^{r} F\left(\mathbb{R}^{N} \times \mathbb{R}^{N}\right)\|=\| g_{1}\right| F_{p q}^{r}\left(\mathbb{R}^{N}\right)\right\|\left\|g_{2} \mid F_{p q}^{r}\left(\mathbb{R}^{N}\right)\right\|
$$

for all $f_{1}, f_{2} \in B_{p q}^{r}\left(\mathbb{R}^{N}\right)$ and $g_{1}, g_{2} \in F_{p q}^{r}\left(\mathbb{R}^{N}\right)$, resp. Note in passing that $\left(\left(R_{12}\right)(f \otimes g)\right)(s, t)=f^{*}(s) g^{*}(t)$ for measurable $f=f(x)$ and $g=g(y)$, $x, y \in \mathbb{R}^{N}$.

\section{THEOREM 4.1.}

(1) Let $0<p<\infty$ and $1<q \leq \infty$. Then

$$
\begin{aligned}
\sup \left\{\left(R_{12} f\right)(s, t): \| f \mid S_{p q}^{N / p} B\left(\mathbb{R}^{N}\right.\right. & \left.\left.\times \mathbb{R}^{N}\right) \| \leq 1\right\} \\
& \sim(\log e / s)^{1 / q^{\prime}}(\log e / t)^{1 / q^{\prime}}
\end{aligned}
$$

for all $0<s, t \leq 1$.

(2) Let $1<p<\infty$ and $0<q \leq \infty$. Then

$$
\begin{aligned}
\sup \left\{\left(R_{12} f\right)(s, t): \| f \mid S_{p q}^{N / p} F\left(\mathbb{R}^{N}\right.\right. & \left.\left.\times \mathbb{R}^{N}\right) \| \leq 1\right\} \\
& \sim(\log e / s)^{1 / p^{\prime}}(\log e / t)^{1 / p^{\prime}}
\end{aligned}
$$

for all $0<s, t \leq 1$.

Proof. One direction follows from (3.2) in case (1) and from (3.2) combined with Corollary 3.2 in case (2).

The converse claim follows using (4.1), (4.2), and tensor products of extremal functions in $B_{p q}^{N / p}\left(\mathbb{R}^{N}\right)$, which were introduced in [ET], [Tri2], and $[\mathrm{H}]$. We do not give details since the proof follows an analogous one in [KS3].

\section{THEOREM 4.2 .}

(1) Let $0<p<\infty$ and $1<q \leq \infty$. If there exists $c>0$ such that

$$
\begin{array}{r}
\int_{0}^{1} \int_{0}^{1}\left[\frac{\left(R_{12} f\right)(s, t)}{(\log e / s)^{1 / q^{\prime}}(\log e / t)^{1 / q^{\prime}}}\right]^{u} \frac{d s}{s \log e / s} \frac{d t}{t \log e / t} \\
\leq c\left\|f \mid S_{p q}^{N / p} B\left(\mathbb{R}^{N} \times \mathbb{R}^{N}\right)\right\|^{u}
\end{array}
$$

for all $f \in S_{p q}^{N / p} B\left(\mathbb{R}^{N} \times \mathbb{R}^{N}\right)$, then $q \leq u$. 
(2) Let $1<p<\infty$ and $0<q \leq \infty$. If there exists $c>0$ such that

$$
\begin{aligned}
& \qquad \begin{array}{l}
\int_{0}^{1} \int_{0}^{1}\left[\frac{\left(R_{12} f\right)(s, t)}{(\log e / s)^{1 / p^{\prime}}(\log e / t)^{1 / p^{\prime}}}\right]^{u} \frac{d s}{s \log e / s} \frac{d t}{t \log e / t} \\
\quad \leq c\left\|f \mid S_{p q}^{N / p} F\left(\mathbb{R}^{N} \times \mathbb{R}^{N}\right)\right\|^{u}
\end{array} \\
& \text { for all } f \in S_{p q}^{N / p} F\left(\mathbb{R}^{N} \times \mathbb{R}^{N}\right) \text {, then } p \leq u
\end{aligned}
$$

The proof is again an appropriate modification of the arguments from Section 5 of [KS3]. One uses tensor products of extremal functions, (4.1) and (4.2).

REMARK 4.3. The left hand side of (4.3) and (4.4) can be considered as a local multivariate growth envelope function in the spirit of [Tri2] or $[\mathrm{H}]$. In this sense Theorems 4.1 and 4.2 establish the local multivariate growth envelopes for the spaces $S_{p q}^{N / p} B\left(\mathbb{R}^{N} \times \mathbb{R}^{N}\right)$ and $S_{p q}^{N / p} F\left(\mathbb{R}^{N} \times \mathbb{R}^{N}\right)$, respectively.

5. The standard case revisited, comparisons. We shall now discuss the behaviour of the normal rearrangement $f^{*}(t)$. Moreover, it is of interest to compare our results with those for isotropic spaces of the same "order of smoothness", close to the spaces with dominating mixed smoothness. While it is clear that for a non-negative integer $r$ the space $W_{p}^{2 r}\left(\mathbb{R}^{2 N}\right)$ is imbedded into $S_{p}^{r} W\left(\mathbb{R}^{2 N}\right)$, the analogous claim for the Besov spaces is not immediate. Nevertheless, such an imbedding is true and we state both imbeddings in the following

Proposition 5.1.

(1) If $1<p<\infty$ and $r \in \mathbb{N}_{0}$, then

$$
W_{p}^{2 r}\left(\mathbb{R}^{2 N}\right) \hookrightarrow S_{p}^{r} W\left(\mathbb{R}^{2 N}\right) .
$$

(2) If $1 \leq p \leq \infty, 0<q \leq \infty$, and $0<r<\infty$, then

$$
B_{p, q}^{2 r}\left(\mathbb{R}^{2 N}\right) \hookrightarrow S_{p q}^{r} B\left(\mathbb{R}^{N} \times \mathbb{R}^{N}\right) .
$$

Proof. As observed above, the statement in (1) is trivial. As to (2) we have - somewhat surprisingly - to dig much deeper. Let $\left\{\varphi_{j}(\xi)\right\}$ be a smooth dyadic resolution of unity in $\mathbb{R}^{N}$ and put

$$
\begin{aligned}
& \psi_{l}(\xi, \eta)=\sum_{\max (j, k)=l} \varphi_{j}(\xi) \varphi_{k}(\eta), \quad l=1,2, \ldots, \\
& \psi_{0}(\xi, \eta)=\varphi_{0}(\xi) \varphi_{0}(\eta) .
\end{aligned}
$$


Then $\sum_{l=0}^{\infty} \psi_{l} \equiv 1$ and $\left\{\psi_{l}\right\}$ is a dyadic resolution of unity in $\mathbb{R}^{2 N}$, subordinated to "dyadic corridors", and we have

$$
\left\|f \mid B_{p, q}^{2 r}\left(\mathbb{R}^{2 N}\right)\right\| \sim\left(\sum_{l=0}^{\infty} 2^{2 r q}\left\|\mathcal{F}^{-1} \psi_{l} \mathcal{F} f \mid L_{p}\left(\mathbb{R}^{2 N}\right)\right\|^{q}\right)^{1 / q} .
$$

Put

$$
\begin{aligned}
& \Psi^{0}=\psi_{0}+\psi_{1}, \\
& \Psi^{l}=\psi_{l-1}+\psi_{l}+\psi_{l+1}, \quad l=1,2, \ldots
\end{aligned}
$$

If $\max (j, k)=l$, then

$$
\varphi_{j}(\xi) \varphi_{k}(\eta)=\varphi_{j}(\xi) \varphi_{k}(\eta) \Psi^{l}(\xi, \eta)
$$

since $\Psi^{l} \equiv 1$ on $\operatorname{supp} \varphi_{j} \otimes \varphi_{k}$. Moreover, if $\max (j, k)=l$, then by Young's inequality,

$$
\begin{aligned}
\| \mathcal{F}^{-1}\left[\varphi_{j}\right. & \left.\otimes \varphi_{k} \mathcal{F} f\right] \mid L_{p}\left(\mathbb{R}^{2 N}\right) \| \\
& =\left\|\mathcal{F}^{-1}\left[\varphi_{j} \otimes \varphi_{k} \mathcal{F}\left(\mathcal{F}^{-1} \Psi^{l} \mathcal{F} f\right)\right] \mid L_{p}\left(\mathbb{R}^{2 N}\right)\right\| \\
& \leq c\left\|\mathcal{F}^{-1}\left(\varphi_{j} \otimes \varphi_{k}\right)\left|L_{1}\left(\mathbb{R}^{2 N}\right)\|\| \mathcal{F}^{-1}\left(\Psi^{l} \mathcal{F} f\right)\right| L_{p}\left(\mathbb{R}^{2 N}\right)\right\| \\
& \leq c\left\|\mathcal{F}^{-1}\left(\Psi^{l} \mathcal{F} f\right) \mid L_{p}\left(\mathbb{R}^{2 N}\right)\right\|,
\end{aligned}
$$

where the constant $c$ is independent of $j$ and $k$. Hence

$$
\begin{aligned}
\| f \mid S_{p q}^{r} B\left(\mathbb{R}^{N} \times\right. & \left.\mathbb{R}^{N}\right)\left\|^{q} \leq \sum_{l=0}^{\infty} \sum_{\max (j, k)=l} 2^{(j+k) r q}\right\| \mathcal{F}^{-1} \varphi_{j} \otimes \varphi_{k} \mathcal{F} f \mid L_{p}\left(\mathbb{R}^{2 N}\right) \|^{q} \\
& \leq c \sum_{l=0}^{\infty}\left\|\mathcal{F}^{-1} \Psi^{l} \mathcal{F} f \mid L_{p}\left(\mathbb{R}^{2 N}\right)\right\|^{q} \sum_{\max (j, k)=l} 2^{(j+k) r q} \\
& \leq c \sum_{l=0}^{\infty}\left\|\mathcal{F}^{-1} \Psi^{l} \mathcal{F} f \mid L_{p}\left(\mathbb{R}^{2 N}\right)\right\|^{q}\left(\sum_{j=0}^{l} 2^{(l+j) r q}+\sum_{k=0}^{l-1} 2^{(l+k) r q}\right) \\
& \leq c \sum_{l=0}^{\infty}\left\|\mathcal{F}^{-1} \Psi^{l} \mathcal{F} f \mid L_{p}\left(\mathbb{R}^{2 N}\right)\right\|^{q}\left(2^{2 l r q+1} \sum_{j=0}^{l} 2^{(j-l) r q}\right)
\end{aligned}
$$

and the last sum on the right hand side is $\leq c$ iff $r>0$, and $\leq c l$ for $r=0$. We conclude that

$$
\begin{aligned}
\left\|f \mid S_{p q}^{r} B\left(\mathbb{R}^{N} \times \mathbb{R}^{N}\right)\right\|^{q} & \leq c \sum_{l=0}^{\infty} 2^{2 r l q}\left\|\mathcal{F}^{-1} \Psi^{l} \mathcal{F} f \mid L_{p}\left(\mathbb{R}^{2 N}\right)\right\|^{q} \\
& \leq c \sum_{l=0}^{\infty} 2^{2 r l q}\left\|\mathcal{F}^{-1} \psi_{l} \mathcal{F} f \mid L_{p}\left(\mathbb{R}^{2 N}\right)\right\|^{q}
\end{aligned}
$$


REMARK 5.2. If $r=0$, then

$$
\begin{aligned}
\left\|f \mid S_{p q}^{0} B\left(\mathbb{R}^{N} \times \mathbb{R}^{N}\right)\right\| & \leq c\left(\sum_{l=0}^{\infty}\left[\left\|(l+1)^{1 / q} \mathcal{F}^{-1} \psi_{l} \mathcal{F} f \mid L_{p}\left(\mathbb{R}^{2 N}\right)\right\|\right]^{q}\right)^{1 / q} \\
& =c\left\|f \mid B_{p, q}^{0,1 / q}\left(\mathbb{R}^{2 N}\right)\right\| ;
\end{aligned}
$$

there is a space with generalized smoothness on the right hand side. For properties of these spaces we refer to $[\mathrm{L}]$.

In the subcritical case the following imbeddings hold:

$$
W_{p}^{2 r}\left(\mathbb{R}^{2 N}\right) \hookrightarrow S_{p}^{r} W\left(\mathbb{R}^{N} \times \mathbb{R}^{N}\right) \hookrightarrow L_{p^{*}}\left(\mathbb{R}^{N} \times \mathbb{R}^{N}\right)
$$

and

$$
B_{p q}^{2 r}\left(\mathbb{R}^{2 N}\right) \hookrightarrow S_{p q}^{r} B\left(\mathbb{R}^{N} \times \mathbb{R}^{N}\right) \hookrightarrow L_{p^{*}}\left(\mathbb{R}^{N} \times \mathbb{R}^{N}\right),
$$

where $1<p<\infty, 0<q \leq \infty$, and

$$
r-\frac{N}{p}=-\frac{N}{p^{*}} \quad\left(\text { that is, } p^{*}=\frac{N p}{N-r p}\right) .
$$

None of these imbeddings can be improved with respect to $p^{*}$. Now we consider the critical case $r=N / p$. Then all spaces under consideration contain unbounded functions and the question of measuring their unboundedness makes sense. This turns out to be different in these cases. It is known from the theory of growth envelopes (see $[\mathrm{H}]$, [Tri2]) that for small $\varepsilon$,

$$
\sup \left\{f^{*}(t):\left\|f \mid W_{p}^{2 N / p}\left(\mathbb{R}^{2 N}\right)\right\| \leq 1\right\} \sim|\log t|^{1 / p^{\prime}}, \quad 0<t<\varepsilon,
$$

and

$$
\int_{0}^{\varepsilon}\left[\frac{f^{*}(t)}{|\log t|}\right]^{p} \frac{d t}{t} \leq c\left\|f \mid W_{p}^{2 N / p}\left(\mathbb{R}^{2 N}\right)\right\|^{p} .
$$

The exponent $p$ on the left hand side is sharp. Moreover, if $0<p<\infty$ and $1<q \leq \infty$, then

$$
\sup \left\{f^{*}(t):\left\|f \mid B_{p, q}^{2 N / p}\left(\mathbb{R}^{2 N}\right)\right\| \leq 1\right\} \sim|\log t|^{1 / q^{\prime}}
$$

and

$$
\int_{0}^{\varepsilon}\left[\frac{f^{*}(t)}{|\log t|}\right]^{q} \frac{d t}{t} \leq c\left\|f \mid B_{p, q}^{2 N / p}\left(\mathbb{R}^{2 N}\right)\right\|^{q},
$$

where again the exponent $q$ on the left hand side is sharp.

THEOREM 5.3.

(1) Let $1<p<\infty$ and let $f \in S_{p}^{N / p} W\left(\mathbb{R}^{2 N}\right)$ be supported in $\Omega_{1} \times \Omega_{2} \subset$ $\mathbb{R}^{N} \times \mathbb{R}^{N},\left|\Omega_{1}\right|=\left|\Omega_{2}\right|=1$. Then

$$
\sup \left\{f^{*}(t):\left\|f \mid S_{p}^{N / p} W\left(\mathbb{R}^{2 N}\right)\right\| \leq 1\right\} \sim\left(\log \frac{e}{t}\right)^{2 / p^{\prime}}
$$


for all $0<t \leq 1$, and

$$
\int_{0}^{1}\left[\frac{f^{*}(t)}{(\log e / t)^{2}}\right]^{p} \frac{d t}{t} \leq c\left\|f \mid S_{p}^{N / p} W\left(\mathbb{R}^{2 N}\right)\right\|^{p} .
$$

Moreover, $S_{p}^{N / p} W$ in (5.6) and (5.7) can be replaced by $S_{p q}^{N / p} F$ with an arbitrary $q, 0<q \leq \infty$.

(2) Let $1 \leq p<\infty, 1<q<\infty$, and let $f \in S_{p q}^{N / p} B\left(\mathbb{R}^{N} \times \mathbb{R}^{N}\right)$ be supported in $\Omega_{1} \times \Omega_{2} \subset \mathbb{R}^{N} \times \mathbb{R}^{N},\left|\Omega_{1}\right|=\left|\Omega_{2}\right|=1$. Then

$$
\sup \left\{f^{*}(t):\left\|f \mid S_{p q}^{N / p} B\left(\mathbb{R}^{N} \times \mathbb{R}^{N}\right)\right\| \leq 1\right\} \sim\left(\log \frac{e}{t}\right)^{2 / q^{\prime}}
$$

for all $0<t \leq 1$, and

$$
\int_{0}^{1}\left[\frac{f^{*}(t)}{(\log e / t)^{2}}\right]^{q} \frac{d t}{t} \leq c\left\|f \mid S_{p q}^{N / p} B\left(\mathbb{R}^{N} \times \mathbb{R}^{N}\right)\right\|^{q} .
$$

Proof. To prove the equivalence in (5.6) and (5.8) we use again the arguments from [KS3, Theorem 5.4] (extremal functions), now in combination with the arguments in Remark 2.7. This gives the estimates of the envelope function from below. The remaining part of (1) follows from Corollaries 3.2, $2.10,2.6$, and 2.3. As to (2) the remaining estimate is a consequence of (3.2), Corollary 2.6, and Corollary 2.3.

REMARK 5.4. We point out the interesting appearance of different exponents: $2 / p^{\prime}$ and $2 / q^{\prime}$ in $(5.6)-(5.9)$, and $1 / p^{\prime}$ and $1 / q^{\prime}$ in $(5.2)-(5.5)$.

\section{References}

[BR] C. Bennett and K. Rudnick, On Lorentz-Zygmund spaces, Dissertationes Math. (Rozprawy Mat.) 175 (1980).

[BS] C. Bennett and R. Sharpley, Interpolation of Operators, Academic Press, Boston, 1988.

[B] A. P. Blozinski, Multivariate rearrangements and Banach function spaces with mixed norms, Trans. Amer. Math. Soc. 263 (1981), 149-167.

[BW] H. Brézis and S. Wainger, A note on limiting cases of Sobolev embeddings and convolution inequalities, Comm. Partial Differential Equations 5 (1980), 773-789.

[CUK] D. Cruz-Uribe, SFO, and M. Krbec, Localization and extrapolation in LorentzOrlicz spaces, in: M. Cwikel et al. (eds.), Function Spaces, Interpolation Theory and Related Topics (Lund, 2000), de Gruyter, Berlin, 2002, 273-283.

[EK] D. E. Edmunds and M. Krbec, On decomposition in exponential Orlicz spaces, Math. Nachr. 213 (2000), 77-88.

[ET] D. E. Edmunds and H. Triebel, Sharp Sobolev embeddings and related Hardy inequalities - the critical case, Math. Nachr. 207 (1999), 79-92.

[HLP] G. H. Hardy, J. E. Littlewood and G. Pólya, Inequalities, Cambridge Univ. Press, Princeton, 1951. 
[H] D. Haroske, Envelopes and Sharp Embeddings of Function Spaces, Chapman \& Hall/CRC Res. Notes Math. 437, Boca Raton, 2007.

[K] V. I. Kolyada, Embeddings of fractional Sobolev spaces and estimates of Fourier transforms, Mat. Sb. 192 (2001), 51-72 (in Russian); English transl.: Sb. Math. 192 (2001), 979-1000.

[KS1] M. Krbec and H.-J. Schmeisser, Limiting imbeddings. The case of missing derivatives, Ricerche Mat. 45 (1996), 423-447.

[KS2] - - - Imbeddings of Brézis-Wainger type. The case of missing derivatives, Proc. Roy. Soc. Edinburgh Sect. A 131 (2001), 1-34.

[KS3] - - - Refined limiting imbeddings for Sobolev spaces of vector-valued functions, J. Funct. Anal. 227 (2005), 372-388.

[L] H.-G. Leopold, Embeddings and entropy numbers in Besov spaces of generalized smoothness, in: Function Spaces: The Fifth Conference, H. Hudzik and L. Skrzypczak (eds.), Lecture Notes in Pure Appl. Math. 213, Dekker, New York, 2000, 323-336.

[M-S] S. J. Montgomery-Smith, Comparison of Orlicz-Lorentz spaces, Studia Math. 103 (1992), 161-189.

[P] F. J. Pérez, Embedding theorems for anisotropic Lipschitz spaces, ibid. 168 (2005), $51-72$.

[R] M. C. Rodriguez Fernández, Über die Spur von Funktionen mit dominierenden gemischten Glattheitseigenschaften auf der Diagonale, Dissertation, Jena, 1997.

[ScSi] H.-J. Schmeisser and W. Sickel, Spaces of functions of mixed smoothness and approximation from hyperbolic crosses, J. Approx. Theory 128 (2004), 115-150.

[ScT] H.-J. Schmeisser and H. Triebel, Topics in Fourier Analysis and Function Spaces, Geest \& Portig, Leipzig, and Wiley, Chichester, 1987.

[SW] E. M. Stein and G. Weiss, Introduction to Fourier Analysis in Euclidean Spaces, Princeton Univ. Press, Princeton, 1971.

[Tri1] H. Triebel, Theory of Function Spaces, Geest \& Portig, Leipzig, and Birkhäuser, Basel, 1983.

[Tri2] - The Structure of Functions, Birkhäuser, Basel, 2001.

[Tru] N. Trudinger, On imbeddings into Orlicz spaces and some applications, J. Math. Mech. 17 (1967), 473-483.

[V] J. Vybíral, Characterization of function spaces with dominating mixed smoothness properties, Jenaer Schriften Math. Inform. Math/Inf/15/03, 1-42.

[Ya] A. A. Yatsenko, Iterative rearrangements of functions and Lorentz spaces, Izv. Vyssh. Ucheb. Zav. Mat. 432 (1998), 73-77 (in Russian).

Institute of Mathematics

Academy of Sciences of the Czech Republic

Žitná 25

11567 Praha 1, Czech Republic

E-mail: krbecm@math.cas.cz
Mathematisches Institut Fakultät für Mathematik und Informatik Friedrich-Schiller-Universität Ernst-Abbe-Platz 1-2 07743 Jena, Germany E-mail: mhj@minet.uni-jena.de

Received April 20, 2006

Revised version May 14, 2007 